Article

\title{
Some Novel Fractional Integral Inequalities over a New Class of Generalized Convex Function
}

\author{
Soubhagya Kumar Sahoo ${ }^{1}\left(\mathbb{D}\right.$, Muhammad Tariq $^{2} \mathbb{D}$, Hijaz Ahmad ${ }^{3,4} \mathbb{D}$, Bibhakar Kodamasingh ${ }^{1}$, \\ Asif Ali Shaikh ${ }^{2}$, Thongchai Botmart ${ }^{5, *(D)}$ and Mohammed A. El-Shorbagy ${ }^{6,7}$ (D)
}

check for updates

Citation: Sahoo, S.K.; Tariq, M.; Ahmad, H.; Kodamasingh, B.; Shaikh, A.A.; Botmart, T.; El-Shorbagy, M.A. Some Novel Fractional Integral Inequalities over a New Class of Generalized Convex Function. Fractal Fract. 2022, 6, 42. https://doi.org/ $10.3390 /$ fractalfract6010042

Academic Editor: Ahmed I. Zayed

Received: 3 December 202

Accepted: 11 January 2022

Published: 13 January 2022

Publisher's Note: MDPI stays neutral with regard to jurisdictional claims in published maps and institutional affiliations.

Copyright: (C) 2022 by the authors. Licensee MDPI, Basel, Switzerland. This article is an open access article distributed under the terms and conditions of the Creative Commons Attribution (CC BY) license (https:// creativecommons.org/licenses/by/ $4.0 /)$.

1 Department of Mathematics, Institute of Technical Education and Research, Siksha 'O' Anusandhan University, Bhubaneswar 751030, India; soubhagyakumarsahoo@soa.ac.in (S.K.S.); bibhakarkodamasingh@soa.ac.in (B.K.)

2 Department of Basic Sciences and Related Studies, Mehran University of Engineering and Technology, Jamshoro 76062, Pakistan; captaintariq2187@gmail.com (M.T.); asif.shaikh@faculty.muet.edu.pk (A.A.S.)

3 Information Technology Application and Research Center, Istanbul Ticaret University, Istanbul 34445, Turkey; hijaz@ticaret.edu.tr or hijaz555@gmail.com

4 Department of Mathematics, Faculty of Humanities and Social Sciences, Istanbul Ticaret University, Istanbul 34445, Turkey

5 Department of Mathematics, Faculty of Science, Khon Kaen University, Khon Kaen 40002, Thailand

6 Department of Mathematics, College of Science and Humanities in Al-Kharj, Prince Sattam Bin Abdulaziz University, Al-Kharj 11942, Saudi Arabia; ma.hassan@psau.edu.sa

7 Department of Basic Engineering Science, Faculty of Engineering, Menoufia University, Shebin El-Kom 32511, Egypt

* Correspondence: thongbo@kku.ac.th

\begin{abstract}
The comprehension of inequalities in convexity is very important for fractional calculus and its effectiveness in many applied sciences. In this article, we handle a novel investigation that depends on the Hermite-Hadamard-type inequalities concerning a monotonic increasing function. The proposed methodology deals with a new class of convexity and related integral and fractional inequalities. There exists a solid connection between fractional operators and convexity because of its fascinating nature in the numerical sciences. Some special cases have also been discussed, and several already-known inequalities have been recaptured to behave well. Some applications related to special means, q-digamma, modified Bessel functions, and matrices are discussed as well. The aftereffects of the plan show that the methodology can be applied directly and is computationally easy to understand and exact. We believe our findings generalise some well-known results in the literature on s-convexity.
\end{abstract}

Keywords: Hermite-Hadamard inequality; fractional integral operator; convex function; $Q$-s-convex function; special means; q-digamma functions; modified Bessel functions; matrices

\section{Introduction}

The theory of convexity is an amazing and compelling methodology for contemplating enormous and beautiful issues that emerge in different fields of pure and applied sciences [1-3]. Numerous new structures have been presented and researched involving convex sets and related functions. This theory has a rich history and has been the focus and motivation for unusual mathematical research for over a century. Numerous speculations, variations, and theories within convexity theory have been drawn into the thoughts of various mathematicians. The theory of convexity, moreover, is accepted as a critical part in the progression of the idea of inequalities. Inequalities have an intriguing mathematical model because of their significant applications in traditional calculus, fractional calculus [4], quantum calculus [5], interval valued [6], stochastic [7], time-scale calculus [8], fractal sets [9], etc. 
Definition 1 ([10]). Let $\mathcal{G}: \mathbb{I} \rightarrow \mathbb{R}$ be a real valued function. A function $\mathcal{G}$ is said to be convex if

$$
\mathcal{G}\left(\kappa \mathrm{b}_{1}+(1-\kappa) \mathrm{b}_{2}\right) \leq \kappa \mathcal{G}\left(\mathrm{b}_{1}\right)+(1-\kappa) \mathcal{G}\left(\mathrm{b}_{2}\right),
$$

holds for all $\mathrm{b}_{1}, \mathrm{~b}_{2} \in \mathbb{I}$ and $\kappa \in[0,1]$.

Indeed, convex mapping is perhaps the most fundamental and significant mapping in the hypothesis of mathematical inequality because it has general applications in pure and applied mathematics [11], mechanics [12], statistics [13], and economics [14]. Recently, speculation, augmentations, variations, and refinements related to convexity have raised legitimate concerns among a few scientists.

Many integral inequalities have been developed so far by different researchers in the due course of time. In the literature, convexity is one of the most important notions involved in proving inequalities and their applications. In the literature, we have many types of inequalities that involve convex functions, such as Hardy-type inequalities [15], Olsen inequalities [16], Gagliardo-Nirenberg-type inequalities [17], Hermite-HadamardFejér-type inequalities [18], Opial inequalities [19], Simpson type inequalities [20], and Ostrowski inequalities [21]. Likewise, there are a lot of well-known integral inequalities but the most notable one is the Hermite-Hadamard-type integral inequality.

Definition 2 ([22]). A function $\mathcal{G}:[0,+\infty) \rightarrow \mathbb{R}$ is called s-convex, in the second sense, if

$$
\mathcal{G}\left(\kappa b_{1}+(1-\kappa) b_{2}\right) \leq \kappa^{s} \mathcal{G}\left(b_{1}\right)+(1-\kappa)^{s} \mathcal{G}\left(b_{2}\right),
$$

holds $\forall \mathrm{b}_{1}, \mathrm{~b}_{2} \in[0,+\infty), s \in(0,1]$ and $\kappa \in[0,1]$.

During the literature review, we noticed that most of the researchers in this field use the notion of convex function to establish different types of integral inequalities. For example, the Hermite-Hadamard inequality for convex functions is defined as follows:

Let $\mathcal{G}:\left[b_{1}, b_{2}\right] \subset \mathbb{R} \longrightarrow \mathbb{R}$ be an integrable convex function with $b_{1}<b_{2}$. Then, the Hermite-Hadamard inequality is expressed as follows: (see [23]):

$$
\mathcal{G}\left(\frac{\mathrm{b}_{1}+\mathrm{b}_{2}}{2}\right) \leq \frac{1}{\mathrm{~b}_{2}-\mathrm{b}_{1}} \int_{\mathrm{b}_{1}}^{\mathrm{b}_{2}} \mathcal{G}(x) d x \leq \frac{\mathcal{G}\left(\mathrm{b}_{1}\right)+\mathcal{G}\left(\mathrm{b}_{2}\right)}{2} .
$$

Later, under the assumption of s-convexity theory, Dragomir and Fitzpatrick [24] established the following version of $\mathrm{H}-\mathrm{H}$ inequality:

$$
2^{s-1} \mathcal{G}\left(\frac{\mathrm{b}_{1}+\mathrm{b}_{2}}{2}\right) \leq \frac{1}{\mathrm{~b}_{2}-\mathrm{b}_{1}} \int_{\mathrm{b}_{1}}^{\mathrm{b}_{2}} \mathcal{G}(x) d x \leq \frac{\mathcal{G}\left(\mathrm{b}_{1}\right)+\mathcal{G}\left(\mathrm{b}_{2}\right)}{s+1} .
$$

Avci et al. [25] presented a new integral identity for differentiable mappings and established related $\mathrm{H}-\mathrm{H}$-type inequalities for mappings whose first derivatives are $s$-convex. Moreover, İşcan [26] took a step in the advancement of this inequality by establishing a new version of the $\mathrm{H}-\mathrm{H}$ inequality via harmonic convex functions. Toplu et al. [27] introduced a new generalised convexity called the n-polynomial convex function and also established its related inequalities. Tariq et al. [28] incorporated the concepts of npolynomial convexity, harmonic convexity, and s-convexity to define a new generalization, namely an n-polynomial harmonically s-type convex function. Tunc et al. [29] refined the concept of convex function to present a new function called tgs-convex function, and employing this new concept, they proved the $\mathrm{H}-\mathrm{H}$ type inequality in both classical as well as fractional integrals. Kadakal et al. [30] investigated the notion of exponential convexity and related integral inequalities.

The $\mathrm{H}-\mathrm{H}$ inequality has been extended in different ways using s-convexity. For example, Khan et al. [31] proved some $\mathrm{H}-\mathrm{H}$-type inequalities for $s$-convex and s-concave functions via both classical and fractional integrals. They also provided some applications 
of their results involving error estimations for trapezoidal formulas. In [32], the authors proved some $\mathrm{H}-\mathrm{H}$-type inequalities for functions, whose absolute values are twice differentiable s-convex functions. Further, Özcan and İşcan [33] investigated some related inequalities by applying Hölder-Işcan inequality and improved power mean inequality. Korus [34] provided refinements of the $\mathrm{H}-\mathrm{H}$ inequality for convex and s-convex function using iterated integrals.

In the last few decades, many mathematicians and research scholars have concentrated their great contributions and attention on the study of this inequality. A few scientists have determined new variations related to convex functions; for instance, see [35-39] and the references cited therein. In addition, it is impressive that convexity offers multiple thoughts and fruitful applications in both pure and applied science.

Classical fractional integral operators are introduced and used to define fractional derivatives. The fractional derivative is known to present its corresponding fractional integral in fractional operators with nonsingular kernels. Fractional integral inequalities are valuable speculations on traditional inequalities. The Hadamard inequality shows the geometric representation of convex functions, which has been investigated by numerous researchers for fractional integral operators. Sarikaya et al. [4] started applying the Riemann-Liouville fractional integral operator to the Hermite-Hadamard inequality using convex functions. After the publication of this article, many mathematicians (see for example [40-44]) started employing the same technique for different types of convexities and various new fractional operators. To acquire detailed and in-depth knowledge about fractional inequalities associated with different convexities, we will allude to the following (see [45-49]).

For our purpose, we need the $\Psi$-R-L fractional integral, defined as follows (see references $[50,51])$.

Definition 3. The left side and right side of $\Psi-R-L$ fractional integral of a function $\mathcal{G}$, associated with a monotonic increasing positive function $\Psi(x)$, whose first order derivative is continuous on $(m, n) \subset \mathbb{R}$ and for $\alpha>0$, are given as

$$
I_{m^{+}}^{\alpha: \Psi} \mathcal{G}(x)=\frac{1}{\Gamma(\alpha)} \int_{m}^{x} \Psi^{\prime}(z)(\Psi(x)-\Psi(z))^{\alpha-1} \mathcal{G}(z) d z,
$$

and

$$
I_{n^{-}}^{\alpha: \Psi} \mathcal{G}(x)=\frac{1}{\Gamma(\alpha)} \int_{x}^{n} \Psi^{\prime}(z)(\Psi(z)-\Psi(x))^{\alpha-1} \mathcal{G}(z) d z
$$

\section{Preliminaries}

Before coming to the main discussion, some of the associated definitions and results have been brought forward. Recently, Wu et al. [52] introduced the following new classes of $\varrho$-convex sets and $\varrho$-convex functions.

Definition 4 . A set $\mathbb{I} \subset \mathbb{R}$ is said to be $Q$-convex set if

$$
\mathcal{M}_{[\varrho]}\left(\mathbf{b}_{1}, \mathbf{b}_{2}\right)=\varrho^{-1}\left(\kappa \varrho\left(\mathbf{b}_{1}\right)+(1-\kappa) \varrho\left(\mathbf{b}_{2}\right)\right) \in \mathbb{I}
$$

for each $\mathrm{b}_{1}, \mathrm{~b}_{2} \in \mathbb{I}, \varrho$ is a strictly monotone and continuous function and $\kappa \in[0,1]$.

Definition 5. A function $\mathcal{G}: \mathbb{I} \subset \mathbb{R} \longrightarrow \mathbb{R}$ is said to be $Q$-convex function if

$$
\mathcal{M}_{[\varrho]}\left(\mathrm{b}_{1}, \mathrm{~b}_{2}\right)=\mathcal{G}\left(\varrho^{-1}\left(\kappa \varrho\left(\mathrm{b}_{1}\right)+(1-\kappa) \varrho\left(\mathrm{b}_{2}\right)\right)\right) \leq \kappa \mathcal{G}\left(\mathrm{b}_{1}\right)+(1-\kappa) \mathcal{G}\left(\mathrm{b}_{2}\right)
$$

for each $\mathrm{b}_{1}, \mathrm{~b}_{2} \in \mathbb{I}, \varrho$ is a strictly monotone and continous function and $\kappa \in[0,1]$.

Recently, Mohammed et al. [53] used this new convex function for a fractional operator to present the following results: 
Theorem 1. Let $\mathcal{G}:\left[\mathrm{b}_{1}, \mathrm{~b}_{2}\right] \subseteq \mathbb{R} \longrightarrow \mathbb{R}$ be an integrable $\varrho$-convex function and $\mathcal{G} \in L^{1}\left(\mathrm{~b}_{1}, \mathrm{~b}_{2}\right)$ with $0 \leq \mathrm{b}_{1}<\mathrm{b}_{2}$. If the function $\varrho$ is increasing and positive on $\left[\mathrm{b}_{1}, \mathrm{~b}_{2}\right]$ and $\varrho^{\prime}(x)$ is continuous on $\left(b_{1}, b_{2}\right)$, then for $\alpha>0$

$\mathcal{G}\left(\varrho^{-1}\left(\frac{\varrho\left(\mathrm{b}_{1}\right)+\varrho\left(\mathrm{b}_{2}\right)}{2}\right)\right) \leq \frac{\Gamma(\alpha+1)}{2\left(\varrho\left(\mathrm{b}_{2}\right)-\varrho\left(\mathrm{b}_{1}\right)\right)^{\alpha}}\left[I_{\mathrm{b}_{1}+}^{\alpha, \varrho} \mathcal{G}\left(\mathrm{b}_{2}\right)+I_{\mathrm{b}_{2}-}^{\alpha, \varrho} \mathcal{G}\left(\mathrm{b}_{1}\right)\right] \leq \frac{\mathcal{G}\left(\mathrm{b}_{1}\right)+\mathcal{G}\left(\mathrm{b}_{2}\right)}{2}$.

Theorem 2. Let $\mathcal{G}:\left[\mathrm{b}_{1}, \mathrm{~b}_{2}\right] \subseteq \mathbb{R} \longrightarrow \mathbb{R}$ be an integrable $Q$-convex function and $\mathcal{G} \in L^{1}\left(\mathrm{~b}_{1}, \mathrm{~b}_{2}\right)$ with $0 \leq \mathrm{b}_{1}<\mathrm{b}_{2}$. If the function $\varrho$ is increasing and positive on $\left[\mathrm{b}_{1}, \mathrm{~b}_{2}\right]$ and $\varrho^{\prime}(x)$ is continuous on $\left(b_{1}, b_{2}\right)$, then for $\alpha>0$ we have:

$$
\begin{aligned}
& \mathcal{G}\left(\varrho^{-1}\left(\frac{\varrho\left(\mathrm{b}_{1}\right)+\varrho\left(\mathrm{b}_{2}\right)}{2}\right)\right) \\
& \leq \frac{2^{\alpha-1} \Gamma(\alpha+1)}{\left(\varrho\left(\mathrm{b}_{2}\right)-\varrho\left(\mathrm{b}_{1}\right)\right)^{\alpha}}\left[I^{\alpha, \varrho}\left(\varrho^{-1}\left(\frac{\varrho\left(\mathrm{b}_{1}\right)+\varrho\left(\mathrm{b}_{2}\right)}{2}\right)\right)^{+} \mathcal{G}\left(\mathrm{b}_{2}\right)+I^{\alpha, \varrho}\left(\varrho^{-1}\left(\frac{\varrho\left(\mathrm{b}_{1}\right)+\varrho\left(\mathrm{b}_{2}\right)}{2}\right)\right)^{-} \mathcal{G}\left(\mathrm{b}_{1}\right)\right] \\
& \leq \frac{\mathcal{G}\left(\mathrm{b}_{1}\right)+\mathcal{G}\left(\mathrm{b}_{2}\right)}{2} .
\end{aligned}
$$

Theorem 3. Let $\mathcal{G}:\left[\mathrm{b}_{1}, \mathrm{~b}_{2}\right] \subseteq \mathbb{R} \longrightarrow \mathbb{R}$ be an integrable $\varrho$-convex function and $\mathcal{G} \in L^{1}\left(\mathrm{~b}_{1}, \mathrm{~b}_{2}\right)$ with $0 \leq \mathrm{b}_{1}<\mathrm{b}_{2}$. If the function $\varrho$ is increasing and positive on $\left[\mathrm{b}_{1}, \mathrm{~b}_{2}\right]$ and $\varrho^{\prime}(x)$ is continuous on $\left(b_{1}, b_{2}\right)$, then for $\alpha>0$

$$
\begin{aligned}
& \frac{\mathcal{G}\left(\mathrm{b}_{1}\right)+\mathcal{G}\left(\mathrm{b}_{2}\right)}{2}-\frac{\Gamma(\alpha+1)}{\left(\left(\varrho\left(\mathrm{b}_{2}\right)-\varrho\left(\mathrm{b}_{1}\right)\right)^{\alpha}\right.}\left[I_{\mathrm{b}_{1}+\varrho}^{\alpha:} \mathcal{G}\left(\mathrm{b}_{2}\right)+I_{\mathrm{b}_{2}{ }^{-}}^{\alpha: \varrho}\right] \\
& =\frac{1}{2\left(\left(\varrho\left(\mathrm{b}_{2}\right)-\varrho\left(\mathrm{b}_{1}\right)\right)^{\alpha}\right.} \int_{\mathrm{b}_{1}}^{\mathrm{b}_{2}}\left(\left(\left(\varrho(z)-\varrho\left(\mathrm{b}_{1}\right)\right)^{\alpha}-\left(\left(\varrho\left(\mathrm{b}_{2}\right)-\varrho(z)\right)^{\alpha}\right) \mathcal{G}^{\prime}(z) d z .\right.\right.
\end{aligned}
$$

Theorem 4. Let $\mathcal{G}:\left[\mathrm{b}_{1}, \mathrm{~b}_{2}\right] \subseteq \mathbb{R} \longrightarrow \mathbb{R}$ be an integrable $\varrho$-convex function and $\mathcal{G} \in L^{1}\left(\mathrm{~b}_{1}, \mathrm{~b}_{2}\right)$ with $0 \leq \mathrm{b}_{1}<\mathrm{b}_{2}$. If the function $\varrho$ is increasing and positive on $\left[\mathrm{b}_{1}, \mathrm{~b}_{2}\right]$ and $\varrho^{\prime}(x)$ is continuous on $\left(\mathrm{b}_{1}, \mathrm{~b}_{2}\right)$, then for $\alpha>0$ we have:

$$
\begin{aligned}
& \frac{2^{\alpha-1} \Gamma \alpha+1}{\left(\varrho\left(\mathrm{b}_{2}\right)-\varrho\left(\mathrm{b}_{1}\right)\right)^{\alpha}}\left[I_{\left(\varrho^{-1}\left(\frac{\rho\left(\mathrm{b}_{1}\right)+\rho\left(\mathrm{b}_{2}\right)}{2}\right)\right)^{+}}^{{ }^{\alpha} \varrho} \mathcal{G}\left(\mathrm{b}_{2}\right)+I_{\left(\varrho^{-1}\left(\frac{\varrho\left(\mathrm{b}_{1}\right)+\varrho\left(\mathrm{b}_{2}\right)}{2}\right)\right)^{-}} \mathcal{G}\left(\mathrm{b}_{1}\right)\right] \\
& -\mathcal{G}\left(\varrho^{-1}\left(\frac{\varrho\left(\mathrm{b}_{1}\right)+\varrho\left(\mathrm{b}_{2}\right)}{2}\right)\right) \\
& =\frac{2^{\alpha-1}}{\left(\varrho\left(\mathrm{b}_{2}\right)-\varrho\left(\mathrm{b}_{1}\right)\right)^{\alpha}}\left[\int_{\varrho^{-1}\left(\frac{\rho\left(\mathrm{b}_{1}\right)+\varrho\left(\mathrm{b}_{2}\right)}{2}\right)}^{\mathrm{b}_{2}}\left(\varrho\left(\mathrm{b}_{2}\right)-\varrho(z)\right)^{\alpha} \mathcal{G}^{\prime}(z) d z\right. \\
& \left.-\int_{\mathrm{b}_{1}}^{\varrho^{-1}\left(\frac{\varrho\left(\mathrm{b}_{1}\right)+\varrho\left(\mathrm{b}_{2}\right)}{2}\right)}\left(\varrho(z)-\varrho\left(\mathrm{b}_{1}\right)\right)^{\alpha} \mathcal{G}^{\prime}(z) d z\right]
\end{aligned}
$$

Furthermore, in [52,53], several inequalities of the Hermite-Hadamard type have been established using the notion of $\varrho$-convexity.

The main aim of this article is to introduce a new class of convex functions and, employing this, we present some Hermite-Hadamard inequalities for both classical and fractional integral operators.

Owing to the aforementioned trend and inspired by the ongoing activities, the rest of this paper is structured as follows: First of all, in Sections 1 and 2, we discuss some preliminary ideas about convexity and the theory of inequality. Next, in Section 3, we define and explore the newly introduced idea of $\varrho$-s-convex functions and some of their special cases. Sections 4 and 5 deal with deriving some new variants of $\mathrm{H}-\mathrm{H}$-type inequalities employing $\varrho$-s-convexity via both classical and fractional integral operators. Applications to 
means, q-digamma, modified Bessel functions, and matrices are demonstrated in Section 6. In Section 7, we present the conclusion of the paper.

As s-convex is a very special type of convexity, it has many applications in inequality theory. Several mathematicians have employed innovative approaches to investigate the $\mathrm{H}-$ $\mathrm{H}$ inequality. To take a step forward in the advancement of inequality theory, we have introduced a new type of $s$-convexity, where the function is associated with another monotonic continuous function. It employs the formula $\mathcal{M}_{[\varrho]}\left(\mathrm{b}_{1}, \mathrm{~b}_{2}\right)=\varrho^{-1}\left(\kappa \varrho\left(\mathrm{b}_{1}\right)+(1-\kappa) \varrho\left(\mathrm{b}_{2}\right)\right)$, which is related to all the power means. Furthermore, we have demonstrated our results through applications to means, matrices, and some special functions. The main motivation for this manuscript is to incorporate the concepts of fractional integral operators with the new convexity.

\section{3. $\varrho$-s-Convex Function}

Definition 6. A function $\mathcal{G}: \mathbb{I} \subset \mathbb{R} \longrightarrow \mathbb{R}$ is said to be $\varrho$-s-convex function iff

$$
\mathcal{G}\left(\varrho^{-1}\left(\kappa \varrho\left(\mathrm{b}_{1}\right)+(1-\kappa) \varrho\left(\mathrm{b}_{2}\right)\right)\right) \leq \kappa^{s} \mathcal{G}\left(\mathrm{b}_{1}\right)+(1-\kappa)^{s} \mathcal{G}\left(\mathrm{b}_{2}\right),
$$

holds for some fixed $s \in(0,1)$ and $\varrho$ is a strictly monotone and continous function and $\kappa \in[0,1]$.

Remark 1. When $\varrho(x)=x$, the inequality given in (5) reduces to s-convex function (2).

Remark 2. When $s=1$, the inequality given in (5) reduces to $\varrho$-convex function (4).

Remark 3. When $\kappa=\frac{1}{2}$, the inequality given in (5) reduces to $\varrho$-s mid point convex function as

$$
\mathcal{G}\left(\varrho^{-1}\left(\frac{\varrho(u)+\varrho(v)}{2}\right)\right) \leq \frac{\mathcal{G}(u)+\mathcal{G}(v)}{2^{s}} .
$$

Some special cases are obtained as follows.

Case-I

When $\varrho(x)=\ln x$, the inequality given in (5) reduces to geometric s-convex function as.

$$
\mathcal{G}\left(\mathrm{b}_{1}{ }^{\kappa} \mathrm{b}_{2}{ }^{1-\kappa}\right) \leq \kappa^{s} \mathcal{G}\left(\mathrm{b}_{1}\right)+(1-\kappa)^{s} \mathcal{G}\left(\mathrm{b}_{2}\right),
$$

for $\mathrm{b}_{1}, \mathrm{~b}_{2} \in \mathbb{I}$ and $\kappa \in[0,1]$.

Case-II

When $\varrho(x)=\frac{1}{x}$, the inequality given in (5) reduces to harmonic s-convex function as

$$
\mathcal{G}\left(\frac{\mathrm{b}_{1} \mathrm{~b}_{2}}{\kappa \mathrm{b}_{2}+(1-\kappa) \mathrm{b}_{1}}\right) \leq \kappa^{s} \mathcal{G}\left(\mathrm{b}_{1}\right)+(1-\kappa)^{s} \mathcal{G}\left(\mathrm{b}_{2}\right),
$$

for $b_{1}, b_{2} \in \mathbb{I}$ and $\kappa \in[0,1]$.

Case-III

When $\varrho(x)=x^{p}$, the inequality given in (5) reduces to p-type s-convex function as

$$
\mathcal{G}\left(\left(\kappa \mathrm{b}_{1}{ }^{p}+(1-\kappa) \mathrm{b}_{2}{ }^{p}\right)^{\frac{1}{p}}\right) \leq \kappa^{s} \mathcal{G}\left(\mathrm{b}_{1}\right)+(1-\kappa)^{s} \mathcal{G}\left(\mathrm{b}_{2}\right),
$$

for $b_{1}, b_{2} \in \mathbb{I}$ and $\kappa \in[0,1]$.

Case-IV

When $\varrho(x)=e^{x}$, the inequality given in (5) reduces to log exponential s-convex function as

$$
\mathcal{G}\left(\ln \left(\kappa e^{\mathrm{b}_{1}}+(1-\kappa) e^{\mathrm{b}_{2}}\right)\right) \leq \kappa^{s} \mathcal{G}\left(\mathrm{b}_{1}\right)+(1-\kappa)^{s} \mathcal{G}\left(\mathrm{b}_{2}\right),
$$

for $b_{1}, b_{2} \in \mathbb{I}$ and $\kappa \in[0,1]$. 


\section{Hermite-Hadamard Type and Related Integral Inequalities}

The principal intention of this research article is to establish some generalized HermiteHadamard-type fractional integral inequalities via the $\varrho$-s convex function (5).

Theorem 5. Let $\mathcal{G}:\left[\mathrm{b}_{1}, \mathrm{~b}_{2}\right] \subseteq \mathbb{R} \longrightarrow \mathbb{R}$ be an integrable $\varrho$-s convex function and $\mathcal{G} \in L^{1}\left(\mathrm{~b}_{1}, \mathrm{~b}_{2}\right)$ with $0 \leq \mathrm{b}_{1}<\mathrm{b}_{2}$. If the function $\varrho$ is increasing and positive on $\left[\mathrm{b}_{1}, \mathrm{~b}_{2}\right]$ and $\varrho^{\prime}(x)$ is continuous on $\left(\mathrm{b}_{1}, \mathrm{~b}_{2}\right)$, then for $\alpha>0$

$$
\mathcal{G}\left(\varrho^{-1}\left(\frac{\varrho\left(\mathrm{b}_{1}\right)+\varrho\left(\mathrm{b}_{2}\right)}{2}\right)\right) \leq \frac{1}{2^{s-1}\left(\varrho\left(\mathrm{b}_{2}\right)-\varrho\left(\mathrm{b}_{1}\right)\right)} \int_{\mathrm{b}_{1}}^{\mathrm{b}_{2}} \mathcal{G}(x) \varrho^{\prime}(x) d x \leq \frac{\mathcal{G}\left(\mathrm{b}_{1}\right)+\mathcal{G}\left(\mathrm{b}_{2}\right)}{2^{s-1}(s+1)} .
$$

Proof. To prove the first inequality of (11), assume that $\mathcal{G}$ is a $\varrho$-s convex function, i.e.,

$$
\mathcal{G}\left(\varrho^{-1}(\kappa \varrho(u)+(1-\kappa) \varrho(v))\right) \leq \kappa^{s} \mathcal{G}(u)+(1-\kappa)^{s} \mathcal{G}(v) .
$$

Setting $\kappa=\frac{1}{2}$, we obtain

$$
\begin{gathered}
\mathcal{G}\left(\varrho^{-1}\left(\frac{\varrho(u)+\varrho(v)}{2}\right)\right) \leq \frac{\mathcal{G}(u)+\mathcal{G}(v)}{2^{s}} . \\
2^{s} \mathcal{G}\left(\varrho^{-1}\left(\frac{\varrho(u)+\varrho(v)}{2}\right)\right) \leq \mathcal{G}(u)+\mathcal{G}(v) .
\end{gathered}
$$

Putting

$u=\varrho^{-1}\left(\kappa \varrho\left(\mathrm{b}_{1}\right)+(1-\kappa) \varrho\left(\mathrm{b}_{2}\right)\right)$ and

$v=\varrho^{-1}\left(\kappa \varrho\left(b_{2}\right)+(1-\kappa) \varrho\left(b_{1}\right)\right)$ in $(12)$, one has

$$
\begin{aligned}
& 2^{s} \mathcal{G}\left(\varrho^{-1}\left(\frac{\varrho\left(\mathbf{b}_{1}\right)+\varrho\left(\mathbf{b}_{2}\right)}{2}\right)\right) \\
& \quad \leq \mathcal{G}\left(\varrho^{-1}\left(\kappa \varrho\left(\mathbf{b}_{1}\right)+(1-\kappa) \varrho\left(\mathbf{b}_{2}\right)\right)\right)+\mathcal{G}\left(\varrho^{-1}\left(\kappa \varrho\left(\mathbf{b}_{2}\right)+(1-\kappa) \varrho\left(\mathbf{b}_{1}\right)\right)\right) .
\end{aligned}
$$

Now, integrating both sides of the last inequality w.r.t to $\kappa$ over $[0,1]$, we get

$$
\begin{aligned}
\mathcal{G}\left(\varrho^{-1}\left(\frac{\varrho\left(\mathrm{b}_{1}\right)+\varrho\left(\mathrm{b}_{2}\right)}{2}\right)\right) & \leq \int_{0}^{1} \mathcal{G}\left(\varrho^{-1}\left(\kappa \varrho\left(\mathrm{b}_{1}\right)+(1-\kappa) \varrho\left(\mathrm{b}_{2}\right)\right)\right) d \kappa \\
& +\int_{0}^{1} \mathcal{G}\left(\varrho^{-1}\left(\kappa \varrho\left(\mathrm{b}_{2}\right)+(1-\kappa) \varrho\left(\mathrm{b}_{1}\right)\right)\right) d \kappa \\
& =\frac{1}{2^{s-1}\left(\varrho\left(\mathrm{b}_{2}-\varrho\left(\mathrm{b}_{1}\right)\right)\right.} \int_{\mathrm{b}_{1}}^{\mathrm{b}_{2}} \mathcal{G}(x) \varrho^{\prime}(x) d x .
\end{aligned}
$$

In this way, the first inequality is proved.

For the proof of the second inequality, we use the definition of $\varrho$-s convex function:

$$
\mathcal{G}\left(\varrho^{-1}\left(\kappa \varrho\left(\mathrm{b}_{2}\right)+(1-\kappa) \varrho\left(\mathrm{b}_{1}\right)\right)\right) \leq \kappa^{s} \mathcal{G}\left(\mathrm{b}_{2}\right)+(1-\kappa)^{s} \mathcal{G}\left(\mathrm{b}_{1}\right) .
$$

Integrating w.r.t to $\kappa$ over $[0,1]$, we get 


$$
\int_{0}^{1} \mathcal{G}\left(\varrho^{-1}\left(\kappa \varrho\left(\mathrm{b}_{2}\right)+(1-\kappa) \varrho\left(\mathrm{b}_{1}\right)\right)\right) d \kappa \leq \mathcal{G}\left(\mathrm{b}_{2}\right) \int_{0}^{1} \kappa^{s} d \kappa+\mathcal{G}\left(\mathrm{b}_{1}\right) \int_{0}^{1}(1-\kappa)^{s} d \kappa
$$

Which leads to

$\frac{1}{\left(\varrho\left(\mathrm{b}_{2}\right)-\varrho\left(\mathrm{b}_{1}\right)\right)} \int_{\mathrm{b}_{1}}^{\mathrm{b}_{2}} \mathcal{G}(x) \varrho^{\prime}(x) d x \leq \frac{\mathcal{G}\left(\mathrm{b}_{1}\right)+\mathcal{G}\left(\mathrm{b}_{2}\right)}{s+1}$.

Consequently gives,

$$
\frac{1}{2^{s-1}\left(\varrho\left(\mathrm{b}_{2}\right)-\varrho\left(\mathrm{b}_{1}\right)\right)} \int_{\mathrm{b}_{1}}^{\mathrm{b}_{2}} \mathcal{G}(x) \varrho^{\prime}(x) d x \leq \frac{\mathcal{G}\left(\mathrm{b}_{1}\right)+\mathcal{G}\left(\mathrm{b}_{2}\right)}{2^{s-1}(s+1)} \text {. }
$$

Upon combining the last two inequalities (13) \& (14), we have the desired result, i.e.,

$$
\mathcal{G}\left(\varrho^{-1}\left(\frac{\varrho\left(\mathrm{b}_{1}\right)+\varrho\left(\mathrm{b}_{2}\right)}{2}\right)\right) \leq \frac{1}{2^{s-1}\left(\varrho\left(\mathrm{b}_{2}\right)-\varrho\left(\mathrm{b}_{1}\right)\right)} \int_{\mathrm{b}_{1}}^{\mathrm{b}_{2}} \mathcal{G}(x) \varrho^{\prime}(x) d x \leq \frac{\mathcal{G}\left(\mathrm{b}_{1}\right)+\mathcal{G}\left(\mathrm{b}_{2}\right)}{2^{s-1}(s+1)} .
$$

Theorem 6. Let $\mathcal{G}, \mathcal{H}: \mathbb{I} \longrightarrow \mathbb{R}$ be two integrable $\varrho$-s convex functions with respect to the function $\varrho$. Then the following inequality holds true:

$$
\begin{aligned}
& \frac{1}{\left(\varrho\left(\mathrm{b}_{2}\right)-\varrho\left(\mathrm{b}_{1}\right)\right)^{2}} \int_{\mathrm{b}_{1}}^{\mathrm{b}_{2}}\left(\varrho\left(\mathrm{b}_{2}\right)-\varrho(x)\right)\left(\mathcal{H}\left(\mathrm{b}_{1}\right) \mathcal{G}(x)+\mathcal{G}\left(\mathrm{b}_{1}\right) \mathcal{H}(x)\right) \varrho^{\prime}(x) d x \\
& \quad+\frac{1}{\left(\varrho\left(\mathrm{b}_{2}\right)-\varrho\left(\mathrm{b}_{1}\right)\right)^{2}} \int_{\mathrm{b}_{1}}^{\mathrm{b}_{2}}\left(\varrho(x)-\varrho\left(\mathrm{b}_{1}\right)\right)\left(\mathcal{H}\left(\mathrm{b}_{2}\right) \mathcal{G}(x)+\mathcal{G}\left(\mathrm{b}_{2}\right) \mathcal{H}(x)\right) \varrho^{\prime}(x) d x \\
& \quad \leq \frac{M\left(\mathrm{~b}_{1}, \mathrm{~b}_{2}\right)}{2 s+1}+N\left(\mathrm{~b}_{1}, \mathrm{~b}_{2}\right)[\beta(s+1, s+1)]+\frac{1}{\varrho\left(\mathrm{b}_{2}\right)-\varrho\left(\mathrm{b}_{1}\right)} \int_{\mathrm{b}_{1}}^{\mathrm{b}_{2}} \mathcal{G}(x) \mathcal{H}(x) \varrho^{\prime}(x) d x .
\end{aligned}
$$

Proof. Using the definition of $\varrho$-s convex functions, we have

$$
\mathcal{G}\left(\varrho^{-1}\left(\kappa \varrho\left(\mathrm{b}_{1}\right)+(1-\kappa) \varrho\left(\mathrm{b}_{2}\right)\right)\right) \leq \kappa^{s} \mathcal{G}\left(\mathrm{b}_{1}\right)+(1-\kappa)^{s} \mathcal{G}\left(\mathrm{b}_{2}\right),
$$

and

$$
\mathcal{H}\left(\varrho^{-1}\left(\kappa \varrho\left(\mathrm{b}_{1}\right)+(1-\kappa) \varrho\left(\mathrm{b}_{2}\right)\right)\right) \leq \kappa^{\varsigma} \mathcal{H}\left(\mathrm{b}_{1}\right)+(1-\kappa)^{\varsigma} \mathcal{H}\left(\mathrm{b}_{2}\right) .
$$

Using $e \leq f$ and $p \leq r$

$$
\Longrightarrow \mathrm{er}+\mathrm{fp} \leq \mathrm{ep}+\mathrm{fr} .
$$

$$
\begin{aligned}
& \mathcal{G}\left(\varrho^{-1}\left(\kappa \varrho\left(\mathrm{b}_{1}\right)+(1-\kappa) \varrho\left(\mathrm{b}_{2}\right)\right)\right)\left[\kappa^{s} \mathcal{H}\left(\mathrm{b}_{1}\right)+(1-\kappa)^{s} \mathcal{H}\left(\mathrm{b}_{2}\right)\right] \\
& +\mathcal{H}\left(\varrho^{-1}\left(\kappa \varrho\left(\mathrm{b}_{1}\right)+(1-\kappa) \varrho\left(\mathrm{b}_{2}\right)\right)\right)\left[\kappa^{s} \mathcal{G}\left(\mathrm{b}_{1}\right)+(1-\kappa)^{s} \mathcal{G}\left(\mathrm{b}_{2}\right)\right] \\
& \leq \mathcal{G}\left(\varrho^{-1}\left(\kappa \varrho\left(\mathrm{b}_{1}\right)+(1-\kappa) \varrho\left(\mathrm{b}_{2}\right)\right)\right) \mathcal{H}\left(\varrho^{-1}\left(\kappa \varrho\left(\mathrm{b}_{1}\right)+(1-\kappa) \varrho\left(\mathrm{b}_{2}\right)\right)\right) \\
& +\left[\kappa^{s} \mathcal{G}\left(\mathrm{b}_{1}\right)+(1-\kappa)^{s} \mathcal{G}\left(\mathrm{b}_{2}\right)\right]\left[\kappa^{s} \mathcal{H}\left(\mathrm{b}_{1}\right)+(1-\kappa)^{s} \mathcal{H}\left(\mathrm{b}_{2}\right)\right] .
\end{aligned}
$$


Integrating both the sides, we obtain

$$
\begin{aligned}
& \mathcal{H}\left(\mathrm{b}_{1}\right) \int_{0}^{1} \kappa^{s} \mathcal{G}\left(\varrho^{-1}\left(\left(\kappa \varrho\left(\mathrm{b}_{1}\right)+(1-\kappa) \varrho\left(\mathrm{b}_{2}\right)\right)\right) d \kappa\right. \\
& +\mathcal{H}\left(\mathrm{b}_{2}\right) \int_{0}^{1}(1-\kappa)^{s} \mathcal{G}\left(\varrho^{-1}\left(\kappa \varrho\left(\mathrm{b}_{1}\right)+(1-\kappa) \varrho\left(\mathrm{b}_{2}\right)\right)\right) d \kappa \\
& +\mathcal{G}\left(\mathrm{b}_{1}\right) \int_{0}^{1} \kappa^{s} \mathcal{H}\left(\varrho^{-1}\left(\left(\kappa \varrho\left(\mathrm{b}_{1}\right)+(1-\kappa) \varrho\left(\mathrm{b}_{2}\right)\right)\right) d \kappa\right. \\
& +\mathcal{G}\left(\mathrm{b}_{2}\right) \int_{0}^{1}(1-\kappa)^{s} \mathcal{H}\left(\varrho^{-1}\left(\kappa \varrho\left(\mathrm{b}_{1}\right)+(1-\kappa) \varrho\left(\mathrm{b}_{2}\right)\right)\right) d \kappa \\
& \leq \mathcal{G}\left(\mathrm{b}_{1}\right) \mathcal{H}\left(\mathrm{b}_{1}\right) \int_{0}^{1} \kappa^{2 s} d \kappa+\mathcal{G}\left(\mathrm{b}_{1}\right) \mathcal{H}\left(\mathrm{b}_{2}\right) \int_{0}^{1} \kappa^{\mathcal{S}}(1-\kappa)^{s} d \kappa \\
& +\mathcal{G}\left(\mathrm{b}_{2}\right) \mathcal{H}\left(\mathrm{b}_{1}\right) \int_{0}^{1} \kappa^{s}(1-\kappa)^{s} d \kappa+\mathcal{G}\left(\mathrm{b}_{2}\right) \mathcal{H}\left(\mathrm{b}_{2}\right) \int_{0}^{1}(1-\kappa)^{2 s} d \kappa
\end{aligned}
$$

By changing the variables, we have

$$
\begin{aligned}
& \int_{0}^{1} \kappa^{s} \mathcal{G}\left(\varrho^{-1}\left(\kappa \varrho\left(\mathrm{b}_{1}\right)+(1-\kappa) \varrho\left(\mathrm{b}_{2}\right)\right)\right) d \kappa \\
& =\frac{1}{\left(\varrho\left(\mathrm{b}_{2}\right)-\varrho\left(\mathrm{b}_{1}\right)\right)^{2}} \int_{\mathrm{b}_{1}}^{\mathrm{b}_{2}}\left(\varrho\left(\mathrm{b}_{2}\right)-\varrho(x)\right) \mathcal{G}(x) \varrho^{\prime}(x) d x
\end{aligned}
$$

and

$$
\begin{aligned}
& \int_{0}^{1}(1-\kappa)^{s} \mathcal{G}\left(\varrho^{-1}\left(\kappa \varrho(a)+(1-\kappa) \varrho\left(\mathrm{b}_{2}\right)\right)\right) d \kappa \\
& =\frac{1}{\left(\varrho\left(\mathrm{b}_{2}\right)-\varrho\left(\mathrm{b}_{1}\right)\right)^{2}} \int_{\mathrm{b}_{1}}^{\mathrm{b}_{2}}\left(\varrho(x)-\varrho\left(\mathrm{b}_{1}\right)\right) \mathcal{G}(x) \varrho^{\prime}(x) d x .
\end{aligned}
$$

Similarly,

$$
\begin{aligned}
& \int_{0}^{1} \kappa^{s} \mathcal{H}\left(\varrho^{-1}\left(\kappa \varrho\left(\mathrm{b}_{1}\right)+(1-\kappa) \varrho\left(\mathrm{b}_{2}\right)\right)\right) d \kappa \\
& =\frac{1}{\left(\varrho\left(\mathrm{b}_{2}\right)-\varrho\left(\mathrm{b}_{1}\right)\right)^{2}} \int_{\mathrm{b}_{1}}^{\mathrm{b}_{2}}\left(\varrho\left(\mathrm{b}_{2}\right)-\varrho(x)\right) \mathcal{H}(x) \varrho^{\prime}(x) d x
\end{aligned}
$$

and

$$
\begin{aligned}
& \int_{0}^{1}(1-\kappa)^{s} \mathcal{H}\left(\varrho^{-1}\left(\kappa \varrho\left(\mathrm{b}_{1}\right)+(1-\kappa) \varrho\left(\mathrm{b}_{2}\right)\right)\right) d \kappa \\
& =\frac{1}{\left(\varrho\left(\mathrm{b}_{2}\right)-\varrho\left(\mathrm{b}_{1}\right)\right)^{2}} \int_{\mathrm{b}_{1}}^{\mathrm{b}_{2}}\left(\varrho(x)-\varrho\left(\mathrm{b}_{1}\right)\right) \mathcal{H}(x) \varrho^{\prime}(x) d x .
\end{aligned}
$$

It is easily verified that

$$
\begin{aligned}
\int_{0}^{1} \kappa^{2 s} d \kappa & =\frac{1}{2 s+1}, \\
\int_{0}^{1} \kappa^{s}(1-\kappa)^{s} d \kappa & =\beta(s+1, s+1),
\end{aligned}
$$

and

$$
\int_{0}^{1}(1-\kappa)^{2 s} d \kappa=\frac{1}{2 s+1}
$$


Taking the above computations into consideration, we get

$$
\begin{aligned}
& \frac{\mathcal{H}\left(\mathrm{b}_{1}\right)}{\left(\varrho\left(\mathrm{b}_{2}\right)-\varrho\left(\mathrm{b}_{1}\right)\right)^{2}} \int_{\mathrm{b}_{1}}^{\mathrm{b}_{2}}\left(\varrho\left(\mathrm{b}_{2}\right)-\varrho(x)\right) \mathcal{G}(x) \varrho^{\prime}(x) d x \\
& +\frac{\mathcal{H}\left(\mathrm{b}_{2}\right)}{\left(\varrho\left(\mathrm{b}_{2}\right)-\varrho\left(\mathrm{b}_{1}\right)\right)^{2}} \int_{\mathrm{b}_{1}}^{\mathrm{b}_{2}}\left(\varrho(x)-\varrho\left(\mathrm{b}_{1}\right)\right) \mathcal{G}(x) \varrho^{\prime}(x) d x \\
& +\frac{\mathcal{G}\left(\mathrm{b}_{1}\right)}{\left(\varrho\left(\mathrm{b}_{2}\right)-\varrho\left(\mathrm{b}_{1}\right)\right)^{2}} \int_{\mathrm{b}_{1}}^{\mathrm{b}_{2}}\left(\varrho\left(\mathrm{b}_{2}\right)-\varrho(x)\right) \mathcal{H}(x) \varrho^{\prime}(x) d x \\
& +\frac{\mathcal{G}\left(\mathrm{b}_{2}\right)}{\left(\varrho\left(\mathrm{b}_{2}\right)-\varrho\left(\mathrm{b}_{1}\right)\right)^{2}} \int_{\mathrm{b}_{1}}^{\mathrm{b}_{2}}\left(\varrho(x)-\varrho\left(\mathrm{b}_{1}\right)\right) \mathcal{H}(x) \varrho^{\prime}(x) d x \\
& \leq \frac{\mathcal{G}\left(\mathrm{b}_{1}\right) \mathcal{H}\left(\mathrm{b}_{1}\right)+\mathcal{G}\left(\mathrm{b}_{2}\right) \mathcal{H}\left(\mathrm{b}_{2}\right)}{2 s+1}+\left[\mathcal{G}\left(\mathrm{b}_{1}\right) \mathcal{H}\left(\mathrm{b}_{2}\right)+\mathcal{G}\left(\mathrm{b}_{2}\right) \mathcal{H}\left(\mathrm{b}_{1}\right)\right] \beta(s+1, s+1) .
\end{aligned}
$$

which implies

$$
\begin{aligned}
& \frac{1}{\left(\varrho\left(\mathrm{b}_{2}\right)-\varrho\left(\mathrm{b}_{1}\right)\right)^{2}} \int_{\mathrm{b}_{1}}^{\mathrm{b}_{2}}\left(\varrho\left(\mathrm{b}_{2}\right)-\varrho(x)\right)\left(\mathcal{H}\left(\mathrm{b}_{1}\right) \mathcal{G}(x)+\mathcal{G}\left(\mathrm{b}_{1}\right) \mathcal{H}(x)\right) \varrho^{\prime}(x) d x \\
& \quad+\frac{1}{\left(\varrho\left(\mathrm{b}_{2}\right)-\varrho\left(\mathrm{b}_{1}\right)\right)^{2}} \int_{\mathrm{b}_{1}}^{\mathrm{b}_{2}}\left(\varrho(x)-\varrho\left(\mathrm{b}_{1}\right)\right)\left(\mathcal{H}\left(\mathrm{b}_{2}\right) \mathcal{G}(x)+\mathcal{G}\left(\mathrm{b}_{2}\right) \mathcal{H}(x)\right) \varrho^{\prime}(x) d x \\
& \leq \frac{M\left(\mathrm{~b}_{1}, \mathrm{~b}_{2}\right)}{2 s+1}+N\left(\mathrm{~b}_{1}, \mathrm{~b}_{2}\right)[\beta(s+1, s+1)]+\frac{1}{\varrho\left(\mathrm{b}_{2}\right)-\varrho\left(\mathrm{b}_{1}\right)} \int_{\mathrm{b}_{1}}^{\mathrm{b}_{2}} \mathcal{G}(x) \mathcal{H}(x) \varrho^{\prime}(x) d x .
\end{aligned}
$$

Theorem 7. Let $\mathcal{G}, \mathcal{H}: \mathbb{I} \longrightarrow \mathbb{R}$ be two integrable $\varrho$-s convex functions with respect to the function Q. Then, the following inequality holds true:

$$
\begin{aligned}
\frac{1}{\varrho\left(\mathrm{b}_{2}\right)-\varrho\left(\mathrm{b}_{1}\right)} \int_{\mathrm{b}_{1}}^{\mathrm{b}_{2}}\left[\mathcal{G}\left(\varrho^{-1}\left(\frac{\varrho\left(\mathrm{b}_{1}\right)+\varrho\left(\mathrm{b}_{2}\right)}{2}\right)\right) \mathcal{H}(x)\right. \\
\left.+\mathcal{H}\left(\varrho^{-1}\left(\frac{\varrho\left(\mathrm{b}_{1}\right)+\varrho\left(\mathrm{b}_{2}\right)}{2}\right)\right) \mathcal{G}(x)\right] \varrho^{\prime}(x) d x \\
\leq \frac{1}{2\left(\varrho\left(\mathrm{b}_{2}\right)-\varrho\left(\mathrm{b}_{1}\right)\right)} \int_{\mathrm{b}_{1}}^{\mathrm{b}_{2}} \mathcal{G}(x) \mathcal{H}(x) \varrho^{\prime}(x) d x \\
+\mathcal{G}\left(\varrho^{-1}\left(\frac{\varrho\left(\mathrm{b}_{1}\right)+\varrho\left(\mathrm{b}_{2}\right)}{2}\right)\right) \mathcal{H}\left(\varrho^{-1}\left(\frac{\varrho\left(\mathrm{b}_{1}\right)+\varrho\left(\mathrm{b}_{2}\right)}{2}\right)\right) \\
+\frac{M\left(\mathrm{~b}_{1}, \mathrm{~b}_{2}\right)}{2} \beta(s+1, s+1)+\frac{N\left(\mathrm{~b}_{1}, \mathrm{~b}_{2}\right)}{2(2 s+1)} .
\end{aligned}
$$

Proof. From the definition of $\varrho$-s convex function, we have

$$
\begin{aligned}
& \mathcal{G}\left(\varrho^{-1}\left(\frac{\varrho\left(\mathrm{b}_{1}\right)+\varrho\left(\mathrm{b}_{2}\right)}{2}\right)\right) \\
= & \mathcal{G}\left(\varrho^{-1}\left(\frac{\varrho\left(\varrho^{-1}\left(\kappa \varrho\left(\mathrm{b}_{1}\right)+(1-\kappa) \varrho\left(\mathrm{b}_{2}\right)\right)\right)+\varrho\left(\varrho^{-1}\left((1-\kappa) \varrho\left(\mathrm{b}_{1}\right)+\kappa \varrho\left(\mathrm{b}_{2}\right)\right)\right)}{2}\right)\right) \\
\leq & \frac{\mathcal{G}\left(\varrho^{-1}\left(\kappa \varrho\left(\mathrm{b}_{1}\right)+(1-\kappa) \varrho(b)\right)\right)+\mathcal{G}\left(\varrho^{-1}\left((1-\kappa) \varrho\left(\mathrm{b}_{1}\right)+\kappa \varrho\left(\mathrm{b}_{2}\right)\right)\right)}{2},
\end{aligned}
$$


and

$$
\begin{aligned}
& \mathcal{H}\left(\varrho^{-1}\left(\frac{\varrho\left(\mathbf{b}_{1}\right)+\varrho\left(\mathrm{b}_{2}\right)}{2}\right)\right) \\
= & \mathcal{H}\left(\varrho^{-1}\left(\frac{\varrho\left(\varrho^{-1}\left(\kappa \varrho\left(\mathrm{b}_{1}\right)+(1-\kappa) \varrho\left(\mathrm{b}_{2}\right)\right)\right)+\varrho\left(\varrho^{-1}(1-\kappa) \varrho\left(\mathrm{b}_{1}\right)+\kappa \varrho\left(\mathrm{b}_{2}\right)\right)}{2}\right)\right) \\
\leq & \frac{\mathcal{H}\left(\varrho^{-1}\left(\kappa \varrho\left(\mathrm{b}_{1}\right)+(1-\kappa) \varrho\left(\mathrm{b}_{2}\right)\right)\right)+\mathcal{H}\left(\varrho^{-1}\left((1-\kappa) \varrho\left(\mathrm{b}_{1}\right)+\kappa \varrho\left(\mathrm{b}_{2}\right)\right)\right)}{2} .
\end{aligned}
$$

Using the same technique as the previous theorem, we have

$$
\begin{aligned}
& \mathcal{G}\left(\varrho^{-1}\left(\frac{\varrho\left(\mathrm{b}_{1}\right)+\varrho\left(\mathrm{b}_{2}\right)}{2}\right)\right) \\
& \times \frac{\mathcal{H}\left(\varrho^{-1}\left(\kappa \varrho\left(\mathbf{b}_{1}\right)+(1-\kappa) \varrho\left(\mathbf{b}_{2}\right)\right)\right)+\mathcal{H}\left(\varrho^{-1}\left((1-\kappa) \varrho\left(\mathbf{b}_{1}\right)+\kappa \varrho\left(\mathbf{b}_{2}\right)\right)\right)}{2} \\
& +\mathcal{H}\left(\varrho^{-1}\left(\frac{\varrho\left(\mathrm{b}_{1}\right)+\varrho\left(\mathrm{b}_{2}\right)}{2}\right)\right) \\
& \times \frac{\mathcal{G}\left(\varrho^{-1}\left(\kappa \varrho\left(\mathbf{b}_{1}\right)+(1-\kappa) \varrho\left(\mathrm{b}_{2}\right)\right)\right)+\mathcal{G}\left(\varrho^{-1}\left((1-\kappa) \varrho\left(\mathrm{b}_{1}\right)+\kappa \varrho\left(\mathrm{b}_{2}\right)\right)\right)}{2} \\
& \leq \mathcal{G}\left(\varrho^{-1}\left(\frac{\varrho\left(\mathrm{b}_{1}\right)+\varrho\left(\mathrm{b}_{2}\right)}{2}\right)\right) \mathcal{H}\left(\varrho^{-1}\left(\frac{\varrho\left(\mathrm{b}_{1}\right)+\varrho\left(\mathrm{b}_{2}\right)}{2}\right)\right) \\
& +\left[\frac{\mathcal{G}\left(\varrho^{-1}\left(\kappa \varrho\left(\mathbf{b}_{1}\right)+(1-\kappa) \varrho\left(\mathrm{b}_{2}\right)\right)\right)+\mathcal{G}\left(\varrho^{-1}\left((1-\kappa) \varrho\left(\mathrm{b}_{1}\right)+\kappa \varrho\left(\mathrm{b}_{2}\right)\right)\right)}{2}\right] \\
& \times\left[\frac{\mathcal{H}\left(\varrho^{-1}\left(\kappa \varrho\left(\mathrm{b}_{1}\right)+(1-\kappa) \varrho\left(\mathrm{b}_{2}\right)\right)\right)+\mathcal{H}\left(\varrho^{-1}\left((1-\kappa) \varrho\left(\mathrm{b}_{1}\right)+\kappa \varrho\left(\mathrm{b}_{2}\right)\right)\right)}{2}\right] \\
& \leq \mathcal{G}\left(\varrho^{-1}\left(\frac{\varrho\left(\mathrm{b}_{1}\right)+\varrho\left(\mathrm{b}_{2}\right)}{2}\right)\right) \mathcal{H}\left(\varrho^{-1}\left(\frac{\varrho\left(\mathrm{b}_{1}\right)+\varrho\left(\mathrm{b}_{2}\right)}{2}\right)\right) \\
& +\frac{1}{4}\left\{\mathcal{G}\left(\varrho^{-1}\left(\kappa \varrho\left(\mathbf{b}_{1}\right)+(1-\kappa) \varrho\left(\mathbf{b}_{2}\right)\right)\right) \mathcal{H}\left(\varrho^{-1}\left(\kappa \varrho\left(\mathbf{b}_{1}\right)+(1-\kappa) \varrho\left(\mathbf{b}_{2}\right)\right)\right)\right. \\
& +\mathcal{G}\left(\varrho^{-1}\left(\kappa \varrho\left(\mathrm{b}_{1}\right)+(1-\kappa) \varrho\left(\mathrm{b}_{2}\right)\right)\right) \mathcal{H}\left(\varrho^{-1}\left((1-\kappa) \varrho\left(\mathrm{b}_{1}\right)+\kappa \varrho\left(\mathrm{b}_{2}\right)\right)\right) \\
& +\mathcal{G}\left(\varrho^{-1}\left((1-\kappa) \varrho\left(\mathrm{b}_{1}\right)+\kappa \varrho\left(\mathrm{b}_{2}\right)\right)\right) \mathcal{H}\left(\varrho^{-1}\left(\kappa \varrho\left(\mathrm{b}_{1}\right)+(1-\kappa) \varrho\left(\mathrm{b}_{2}\right)\right)\right) \\
& \left.+\mathcal{G}\left(\varrho^{-1}\left((1-\kappa) \varrho\left(\mathrm{b}_{1}\right)+\kappa \varrho\left(\mathrm{b}_{2}\right)\right)\right) \mathcal{H}\left(\varrho^{-1}\left((1-\kappa) \varrho\left(\mathrm{b}_{1}\right)+\kappa \varrho\left(\mathrm{b}_{2}\right)\right)\right)\right\} \\
& \leq \mathcal{G}\left(\varrho^{-1}\left(\frac{\varrho\left(\mathbf{b}_{1}\right)+\varrho\left(\mathbf{b}_{2}\right)}{2}\right)\right) \mathcal{H}\left(\varrho^{-1}\left(\frac{\varrho\left(\mathbf{b}_{1}\right)+\varrho\left(\mathbf{b}_{2}\right)}{2}\right)\right) \\
& +\frac{1}{4}\left\{\mathcal{G}\left(\varrho^{-1}\left(\kappa \varrho\left(\mathbf{b}_{1}\right)+(1-\kappa) \varrho\left(\mathbf{b}_{2}\right)\right)\right) \mathcal{H}\left(\varrho^{-1}\left(\kappa \varrho\left(\mathbf{b}_{1}\right)+(1-\kappa) \varrho\left(\mathbf{b}_{2}\right)\right)\right)\right. \\
& +\mathcal{G}\left(\varrho^{-1}\left((1-\kappa) \varrho\left(\mathrm{b}_{1}\right)+\kappa \varrho\left(\mathrm{b}_{2}\right)\right)\right) \mathcal{H}\left(\varrho^{-1}\left((1-\kappa) \varrho\left(\mathrm{b}_{1}\right)+\kappa \varrho\left(\mathrm{b}_{2}\right)\right)\right) \\
& +\left(\kappa^{s} \mathcal{G}(a)+(1-\kappa)^{s} \mathcal{G}(b)\right)\left((1-\kappa)^{s} \mathcal{H}(a)+\kappa^{s} \mathcal{H}(b)\right) \\
& \left.+\left((1-\kappa)^{s} \mathcal{G}(a)+\kappa^{s} \mathcal{G}(b)\right)\left(\kappa^{s} \mathcal{H}(a)+(1-\kappa)^{s} \mathcal{H}(b)\right)\right\} \\
& =\mathcal{G}\left(\varrho^{-1}\left(\frac{\varrho\left(\mathbf{b}_{1}\right)+\varrho\left(\mathrm{b}_{2}\right)}{2}\right)\right) \mathcal{H}\left(\varrho^{-1}\left(\frac{\varrho\left(\mathrm{b}_{1}\right)+\varrho\left(\mathrm{b}_{2}\right)}{2}\right)\right) \\
& +\frac{1}{4}\left\{\mathcal{G}\left(\varrho^{-1}\left(\kappa \varrho\left(\mathbf{b}_{1}\right)+(1-\kappa) \varrho\left(\mathbf{b}_{2}\right)\right)\right) \mathcal{H}\left(\varrho^{-1}\left(\kappa \varrho\left(\mathbf{b}_{1}\right)+(1-\kappa) \varrho\left(\mathbf{b}_{2}\right)\right)\right)\right. \\
& +\mathcal{G}\left(\varrho^{-1}\left((1-\kappa) \varrho\left(\mathbf{b}_{1}\right)+\kappa \varrho\left(\mathbf{b}_{2}\right)\right)\right) \mathcal{H}\left(\varrho^{-1}\left((1-\kappa) \varrho\left(\mathbf{b}_{1}\right)+\kappa \varrho\left(\mathbf{b}_{2}\right)\right)\right) \\
& \left.+2 \kappa^{s}(1-\kappa)^{s}[\mathcal{G}(a) \mathcal{H}(a)+\mathcal{G}(b) \mathcal{H}(b)]+\left(\kappa^{2 s}+(1-\kappa)^{2 s}\right)[\mathcal{G}(a) \mathcal{H}(b)+\mathcal{G}(a) \mathcal{H}(b)]\right\} .
\end{aligned}
$$


Now, integrating both the sides,

$$
\begin{aligned}
& \int_{0}^{1} \mathcal{G}\left(\varrho^{-1}\left(\frac{\varrho\left(\mathrm{b}_{1}\right)+\varrho\left(\mathrm{b}_{2}\right)}{2}\right)\right) \\
& \times \frac{\mathcal{H}\left(\varrho^{-1}\left(\kappa \varrho\left(\mathrm{b}_{1}\right)+(1-\kappa) \varrho\left(\mathrm{b}_{2}\right)\right)\right)+\mathcal{H}\left(\varrho^{-1}\left((1-\kappa) \varrho\left(\mathrm{b}_{1}\right)+\kappa \varrho\left(\mathrm{b}_{2}\right)\right)\right)}{2} d \kappa \\
& +\int_{0}^{1} \mathcal{H}\left(\varrho^{-1}\left(\frac{\varrho\left(\mathrm{b}_{1}\right)+\varrho\left(\mathrm{b}_{2}\right)}{2}\right)\right) \\
& \times \frac{\mathcal{G}\left(\varrho^{-1}\left(\kappa \varrho\left(\mathrm{b}_{1}\right)+(1-\kappa) \varrho\left(\mathrm{b}_{2}\right)\right)\right)+\mathcal{G}\left(\varrho^{-1}\left((1-\kappa) \varrho\left(\mathrm{b}_{1}\right)+\kappa \varrho\left(\mathrm{b}_{2}\right)\right)\right)}{2} d \kappa \\
& \leq \mathcal{G}\left(\varrho^{-1}\left(\frac{\varrho\left(\mathrm{b}_{1}\right)+\varrho\left(\mathrm{b}_{2}\right)}{2}\right)\right) \mathcal{H}\left(\varrho^{-1}\left(\frac{\varrho\left(\mathrm{b}_{1}\right)+\varrho\left(\mathrm{b}_{2}\right)}{2}\right)\right) \\
& +\frac{1}{2}\left[\frac{1}{\left(\varrho\left(\mathrm{b}_{2}\right)-\varrho\left(\mathrm{b}_{1}\right)\right)^{2}} \int_{\mathrm{b}_{1}}^{\mathrm{b}_{2}} \mathcal{G}(x) \mathcal{H}(x) \varrho^{\prime}(x) d x+M\left(\mathrm{~b}_{1}, \mathrm{~b}_{2}\right) \beta(s+1, s+1)+\frac{N\left(\mathrm{~b}_{1}, \mathrm{~b}_{2}\right)}{2 s+1}\right] .
\end{aligned}
$$

This implies,

$$
\begin{aligned}
\frac{1}{\varrho\left(\mathrm{b}_{2}\right)-\varrho\left(\mathrm{b}_{1}\right)} \int_{\mathrm{b}_{1}}^{\mathrm{b}_{2}}\left[\mathcal{G}\left(\varrho^{-1}\left(\frac{\varrho\left(\mathrm{b}_{1}\right)+\varrho\left(\mathrm{b}_{2}\right)}{2}\right)\right) \mathcal{H}(x)\right. \\
\left.+\mathcal{H}\left(\varrho^{-1}\left(\frac{\varrho\left(\mathrm{b}_{1}\right)+\varrho\left(\mathrm{b}_{2}\right)}{2}\right)\right) \mathcal{G}(x)\right] \varrho^{\prime}(x) d x \\
\leq \frac{1}{2\left(\varrho\left(\mathrm{b}_{2}\right)-\varrho\left(\mathrm{b}_{1}\right)\right)} \int_{\mathrm{b}_{1}}^{\mathrm{b}_{2}} \mathcal{G}(x) \mathcal{H}(x) \varrho^{\prime}(x) d x \\
+\mathcal{G}\left(\varrho^{-1}\left(\frac{\varrho\left(\mathrm{b}_{1}\right)+\varrho\left(\mathrm{b}_{2}\right)}{2}\right)\right) \mathcal{H}\left(\varrho^{-1}\left(\frac{\varrho\left(\mathrm{b}_{1}\right)+\varrho\left(\mathrm{b}_{2}\right)}{2}\right)\right) \\
+\frac{M\left(\mathrm{~b}_{1}, \mathrm{~b}_{2}\right)}{2} \beta(s+1, s+1)+\frac{N\left(\mathrm{~b}_{1}, \mathrm{~b}_{2}\right)}{2(2 s+1)} .
\end{aligned}
$$

This completes the desired proof.

\section{Fractional Inequalities for $\varrho$-s Convex Function}

Theorem 8. Let $\mathcal{G}:\left[\mathrm{b}_{1}, \mathrm{~b}_{2}\right] \subseteq \mathbb{R} \longrightarrow \mathbb{R}$ be an integrable $\varrho$-s convex function and $\mathcal{G} \in L^{1}\left(\mathrm{~b}_{1}, \mathrm{~b}_{2}\right)$ with $0 \leq \mathrm{b}_{1}<\mathrm{b}_{2}$. If the function $\varrho$ is increasing and positive on $\left[\mathrm{b}_{1}, \mathrm{~b}_{2}\right]$ and $\varrho^{\prime}(x)$ is continuous on $\left(b_{1}, b_{2}\right)$, then for $\alpha>0$

$$
\begin{aligned}
\mathcal{G}\left(\varrho^{-1}\left(\frac{\varrho\left(\mathrm{b}_{1}\right)+\varrho\left(\mathrm{b}_{2}\right)}{2}\right)\right) \leq \frac{\Gamma(\alpha+1)}{2^{s}\left(\varrho\left(\mathrm{b}_{2}\right)-\varrho\left(\mathrm{b}_{1}\right)\right)^{\alpha}}\left[I_{\mathrm{b}_{1}{ }^{\alpha}}^{\alpha, \varrho} \mathcal{G}\left(\mathrm{b}_{2}\right)+I_{\mathrm{b}_{2}{ }^{\alpha, \varrho}} \mathcal{G}\left(\mathrm{b}_{1}\right)\right] \\
\leq \frac{\alpha\left[\mathcal{G}\left(\mathrm{b}_{1}\right)+\mathcal{G}\left(\mathrm{b}_{2}\right)\right]}{2^{s}}\left[\frac{1}{s+\alpha}+\beta(\alpha, s+1)\right] .
\end{aligned}
$$

Proof. To prove the first inequality of (15), assume $\mathcal{G}$ as a $\varrho$-s convex function, i.e.,

$$
\mathcal{G}\left(\varrho^{-1}(\kappa \varrho(u)+(1-\kappa) \varrho(v))\right) \leq \kappa^{s} \mathcal{G}(u)+(1-\kappa)^{s} \mathcal{G}(v) .
$$

Setting $\kappa=\frac{1}{2}$, we obtain

$$
\begin{gathered}
\mathcal{G}\left(\varrho^{-1}\left(\frac{\varrho(u)+\varrho(v)}{2}\right)\right) \leq \frac{\mathcal{G}(u)+\mathcal{G}(v)}{2^{s}} . \\
2^{s} \mathcal{G}\left(\varrho^{-1}\left(\frac{\varrho(u)+\varrho(v)}{2}\right)\right) \leq \mathcal{G}(u)+\mathcal{G}(v) .
\end{gathered}
$$


Putting

$u=\varrho^{-1}\left(\kappa \varrho\left(\mathrm{b}_{1}\right)+(1-\kappa) \varrho\left(\mathrm{b}_{2}\right)\right)$ and

$v=\varrho^{-1}\left(\kappa \varrho\left(\mathrm{b}_{2}\right)+(1-\kappa) \varrho\left(\mathrm{b}_{1}\right)\right)$ in $(16)$, one has

$$
\begin{aligned}
& 2^{s} \mathcal{G}\left(\varrho^{-1}\left(\frac{\varrho\left(\mathbf{b}_{1}\right)+\varrho\left(\mathbf{b}_{2}\right)}{2}\right)\right) \\
& \leq \mathcal{G}\left(\varrho^{-1}\left(\kappa \varrho\left(\mathbf{b}_{1}\right)+(1-\kappa) \varrho\left(\mathbf{b}_{2}\right)\right)\right)+\mathcal{G}\left(\varrho^{-1}\left(\kappa \varrho\left(\mathbf{b}_{2}\right)+(1-\kappa) \varrho\left(\mathbf{b}_{1}\right)\right)\right) .
\end{aligned}
$$

Multiplying both the sides by $\kappa^{\alpha-1}$ and integrating w.r.t to $\kappa$ over $[0,1]$, we get

$$
\begin{aligned}
\frac{2^{s}}{\alpha} \mathcal{G}\left(\varrho^{-1}\left(\frac{\varrho\left(\mathrm{b}_{1}\right)+\varrho\left(\mathrm{b}_{2}\right)}{2}\right)\right) & \leq \int_{0}^{1} \mathcal{G}\left(\varrho^{-1}\left(\kappa \varrho\left(\mathrm{b}_{1}\right)+(1-\kappa) \varrho\left(\mathrm{b}_{2}\right)\right)\right) \kappa^{\alpha-1} d \kappa \\
& +\int_{0}^{1} \mathcal{G}\left(\varrho^{-1}\left(\kappa \varrho\left(\mathrm{b}_{2}\right)+(1-\kappa) \varrho\left(\mathrm{b}_{1}\right)\right)\right) \kappa^{\alpha-1} d \kappa
\end{aligned}
$$

By changing the variables as

$x=\varrho^{-1}\left(\kappa \varrho\left(\mathbf{b}_{1}\right)+(1-\kappa) \varrho\left(\mathbf{b}_{2}\right)\right)$ and $y=\varrho^{-1}\left(\kappa \varrho\left(\mathbf{b}_{2}\right)+(1-\kappa) \varrho\left(\mathbf{b}_{1}\right)\right)$

$$
\begin{aligned}
\frac{2^{s}}{\alpha} \mathcal{G}\left(\varrho^{-1}\left(\frac{\varrho\left(\mathrm{b}_{1}\right)+\varrho\left(\mathrm{b}_{2}\right)}{2}\right)\right) \leq & \frac{1}{\left(\varrho\left(\mathrm{b}_{2}\right)-\varrho\left(\mathrm{b}_{1}\right)\right)^{\alpha}} \int_{\mathrm{b}_{1}}^{\mathrm{b}_{2}} \varrho^{\prime}(x)\left(\varrho\left(\mathrm{b}_{2}\right)-\varrho(x)\right)^{\alpha-1} \mathcal{G}(x) d x \\
& +\frac{1}{\left(\varrho\left(\mathrm{b}_{2}\right)-\varrho\left(\mathrm{b}_{1}\right)\right)^{\alpha}} \int_{\mathrm{b}_{1}}^{\mathrm{b}_{2}} \varrho^{\prime}(y)\left(\varrho(y)-\varrho\left(\mathrm{b}_{1}\right)\right)^{\alpha-1} \mathcal{G}(y) d y .
\end{aligned}
$$

i.e., $\quad \mathcal{G}\left(\varrho^{-1}\left(\frac{\varrho\left(\mathrm{b}_{1}\right)+\varrho\left(\mathrm{b}_{2}\right)}{2}\right)\right) \leq \frac{\Gamma(\alpha+1)}{2^{s}\left(\varrho\left(\mathrm{b}_{2}\right)-\varrho\left(\mathrm{b}_{1}\right)\right)^{\alpha}}\left[I_{\mathrm{b}_{1}{ }^{+}}^{\alpha, \rho} \mathcal{G}\left(\mathrm{b}_{2}\right)+I_{\mathrm{b}_{2}-}^{\alpha, \varrho} \mathcal{G}\left(\mathrm{b}_{1}\right)\right]$.

In this way, the first inequality is proved.

Next, to prove the second inequality, we use the definition of $\varrho$-s convex function:

$$
\mathcal{G}\left(\varrho^{-1}\left(\kappa \varrho\left(\mathrm{b}_{1}\right)+(1-\kappa) \varrho\left(\mathrm{b}_{2}\right)\right)\right) \leq \kappa^{s} \mathcal{G}\left(\mathrm{b}_{1}\right)+(1-\kappa)^{s} \mathcal{G}\left(\mathrm{b}_{2}\right),
$$

and

$$
\mathcal{G}\left(\varrho^{-1}\left(\kappa \varrho\left(\mathrm{b}_{2}\right)+(1-\kappa) \varrho\left(\mathrm{b}_{1}\right)\right)\right) \leq \kappa^{s} \mathcal{G}\left(\mathrm{b}_{2}\right)+(1-\kappa)^{s} \mathcal{G}\left(\mathrm{b}_{1}\right) .
$$

Adding, we get:

$$
\begin{aligned}
& \mathcal{G}\left(\varrho^{-1}\left(\kappa \varrho\left(\mathrm{b}_{1}\right)+(1-\kappa) \varrho\left(\mathrm{b}_{2}\right)\right)\right)+\mathcal{G}\left(\varrho^{-1}\left(\kappa \varrho\left(\mathrm{b}_{2}\right)+(1-\kappa) \varrho\left(\mathrm{b}_{1}\right)\right)\right) \\
& \leq\left[\kappa^{s}+(1-\kappa)^{s}\right]\left[\mathcal{G}\left(\mathrm{b}_{1}\right)+\mathcal{G}\left(\mathrm{b}_{2}\right)\right] .
\end{aligned}
$$

Multiplying both the sides by $\kappa^{\alpha-1}$ and integrating with respect to $\kappa$ over $[0,1]$, we get

$$
\begin{aligned}
& \int_{0}^{1} \mathcal{G}\left(\varrho^{-1}\left(\kappa \varrho\left(\mathbf{b}_{1}\right)+(1-\kappa) \varrho\left(\mathbf{b}_{2}\right)\right)\right) \kappa^{\alpha-1} d \kappa+\int_{0}^{1} \mathcal{G}\left(\varrho^{-1}\left(\kappa \varrho\left(\mathbf{b}_{2}\right)+(1-\kappa) \varrho\left(\mathbf{b}_{1}\right)\right)\right) \kappa^{\alpha-1} d \kappa \\
& \leq\left[\mathcal{G}\left(\mathbf{b}_{1}\right)+\mathcal{G}\left(\mathbf{b}_{2}\right)\right] \int_{0}^{1}\left[\kappa^{\mathcal{S}}+(1-\kappa)^{S}\right] \kappa^{\alpha-1} d \kappa .
\end{aligned}
$$

$$
\frac{\Gamma(\alpha)}{\left(\varrho\left(\mathrm{b}_{2}\right)-\varrho\left(\mathrm{b}_{1}\right)\right)^{\alpha}}\left[I_{\mathrm{b}_{1}}^{\alpha, \varrho} \mathcal{G}\left(\mathrm{b}_{2}\right)+I_{\mathrm{b}_{2}-}^{\alpha, \varrho} \mathcal{G}\left(\mathrm{b}_{1}\right)\right] \leq\left[\mathcal{G}\left(\mathrm{b}_{1}\right)+\mathcal{G}\left(\mathrm{b}_{2}\right)\right]\left[\frac{1}{s+\alpha}+\beta(\alpha, s+1)\right] .
$$

So,

$$
\frac{\Gamma(\alpha+1)}{2^{s}\left(\varrho\left(\mathrm{b}_{2}\right)-\varrho\left(\mathrm{b}_{1}\right)\right)^{\alpha}}\left[I_{\mathrm{b}_{1}+{ }^{\alpha, \rho}} \mathcal{G}\left(\mathrm{b}_{2}\right)+I_{\mathrm{b}_{2}-}^{\alpha, \varrho} \mathcal{G}\left(\mathrm{b}_{1}\right)\right] \leq \frac{\alpha\left[\mathcal{G}\left(\mathrm{b}_{1}\right)+\mathcal{G}\left(\mathrm{b}_{2}\right)\right]}{2^{s}}\left[\frac{1}{s+\alpha}+\beta(\alpha, s+1)\right] .
$$


Combining (17) and (18), we get the desired result.

$$
\begin{array}{r}
\mathcal{G}\left(\varrho^{-1}\left(\frac{\varrho\left(\mathrm{b}_{1}\right)+\varrho\left(\mathrm{b}_{2}\right)}{2}\right)\right) \leq \frac{\Gamma(\alpha+1)}{2^{s}\left(\varrho\left(\mathrm{b}_{2}\right)-\varrho\left(\mathrm{b}_{1}\right)\right)^{\alpha}}\left[I_{{ }_{b_{1}}{ }^{\alpha, \rho}} \mathcal{G}\left(\mathrm{b}_{2}\right)+I_{\mathrm{b}_{2}-}^{\alpha, \rho} \mathcal{G}\left(\mathrm{b}_{1}\right)\right] \\
\leq \frac{\alpha\left[\mathcal{G}\left(\mathrm{b}_{1}\right)+\mathcal{G}\left(\mathrm{b}_{2}\right)\right]}{2^{s}}\left[\frac{1}{s+\alpha}+\beta(\alpha, s+1)\right] .
\end{array}
$$

Remark 4. Under the assumptions of Theorem 8 when $s=1$, we get

$$
\mathcal{G}\left(\varrho^{-1}\left(\frac{\varrho\left(\mathbf{b}_{1}\right)+\varrho\left(\mathrm{b}_{2}\right)}{2}\right)\right) \leq \frac{\Gamma(\alpha+1)}{2\left(\varrho\left(\mathrm{b}_{2}\right)-\varrho\left(\mathrm{b}_{1}\right)\right)^{\alpha}}\left[I_{\mathrm{b}_{1}}^{\alpha, \rho} \mathcal{G}\left(\mathrm{b}_{2}\right)+I_{\mathrm{b}_{2}}^{\alpha, \varrho} \mathcal{G}\left(\mathrm{b}_{1}\right)\right] \leq \frac{\left[\mathcal{G}\left(\mathrm{b}_{1}\right)+\mathcal{G}\left(\mathrm{b}_{2}\right)\right]}{2},
$$

which was established by Mohammed et al. [53].

Remark 5. Under the assumptions of Theorem 8 when $\alpha=1$, we have

$$
\begin{aligned}
\mathcal{G}\left(\varrho^{-1}\left(\frac{\varrho\left(\mathrm{b}_{1}\right)+\varrho\left(\mathrm{b}_{2}\right)}{2}\right)\right) \leq \frac{1}{2^{s-1}\left(\varrho\left(\mathrm{b}_{2}\right)-\varrho\left(\mathrm{b}_{1}\right)\right)} \int_{\mathrm{b}_{1}}^{\mathrm{b}_{2}} \mathcal{G}(x) \varrho^{\prime}(x) d x \\
\leq \frac{\left[\mathcal{G}\left(\mathrm{b}_{1}\right)+\mathcal{G}\left(\mathrm{b}_{2}\right)\right]}{2^{s}}\left[\frac{1}{s+1}+\beta(1, s+1)\right] .
\end{aligned}
$$

Remark 6. Under the assumptions of Theorem 8 when $\varrho(x)=x$, we have the following fractional inequality

$$
\begin{aligned}
& 2^{s-1} \mathcal{G}\left(\frac{\mathrm{b}_{1}+\mathrm{b}_{2}}{2}\right) \\
& \leq \frac{\Gamma(\alpha+1)}{\left(\mathrm{b}_{2}-\mathrm{b}_{1}\right)^{\alpha}}\left[\frac{I_{\mathrm{b}_{1}}^{\alpha} \mathcal{G}\left(\mathrm{b}_{2}\right)+I_{\mathrm{b}_{2}-}^{\alpha} \mathcal{G}\left(\mathrm{b}_{1}\right)}{2}\right] \leq \alpha\left[\frac{\mathcal{G}\left(\mathrm{b}_{1}\right)+\mathcal{G}\left(\mathrm{b}_{2}\right)}{2}\right]\left[\frac{1}{s+\alpha}+\beta(\alpha, s+1)\right] .
\end{aligned}
$$

Remark 7. Under the assumptions of Theorem 8 when $\varrho(x)=x$ and $\alpha=1$, we get

$$
2^{s-1} \mathcal{G}\left(\frac{\mathrm{b}_{1}+\mathrm{b}_{2}}{2}\right) \leq \frac{1}{\mathrm{~b}_{2}-\mathrm{b}_{1}} \int_{\mathrm{b}_{1}}^{\mathrm{b}_{2}} \mathcal{G}(x) d x \leq \frac{\mathcal{G}\left(\mathrm{b}_{1}\right)+\mathcal{G}\left(\mathrm{b}_{2}\right)}{s+1},
$$

which is the classical Hermite-Hadamard inequality under s-convex function proved by Dragomir and Fitzpatrick [24].

Theorem 9. Let $\mathcal{G}:\left[\mathrm{b}_{1}, \mathrm{~b}_{2}\right] \subseteq \mathbb{R} \longrightarrow \mathbb{R}$ be an integrable $\varrho-$ s-convex function and $\mathcal{G} \in$ $L^{1}\left(\mathrm{~b}_{1}, \mathrm{~b}_{2}\right)$ with $0 \leq \mathrm{b}_{1}<\mathrm{b}_{2}$. If the function $\varrho$ is increasing and positive on $\left[\mathrm{b}_{1}, \mathrm{~b}_{2}\right]$ and $\varrho^{\prime}(x)$ is continuous on $\left(b_{1}, b_{2}\right)$, then for $\alpha>0$ we have:

$$
\begin{aligned}
& \mathcal{G}\left(\varrho^{-1}\left(\frac{\varrho\left(\mathrm{b}_{1}\right)+\varrho\left(\mathrm{b}_{2}\right)}{2}\right)\right) \\
& \left.\leq \frac{2^{\alpha-s} \Gamma(\alpha+1)}{\left(\varrho\left(\mathrm{b}_{2}\right)-\varrho\left(\mathrm{b}_{1}\right)\right)^{\alpha}}\left[I_{\left(\varrho^{\alpha}\left(\frac{\rho\left(\mathrm{b}_{1}\right)+\rho\left(\mathrm{b}_{2}\right)}{2}\right)\right)}\right)^{+} \mathcal{G}\left(\mathrm{b}_{2}\right)+I_{\left(\varrho^{\alpha: \varrho}\right.}^{\left.\left(\frac{\rho\left(\mathrm{b}_{1}\right)+\rho\left(\mathrm{b}_{2}\right)}{2}\right)\right)^{-}} \mathcal{G}\left(\mathrm{b}_{1}\right)\right] \\
& \leq\left[\frac{1}{2^{s}(s+\alpha)}+2^{\alpha} \beta_{\frac{1}{2}}(\alpha, s+1)\right] \frac{\alpha\left[\mathcal{G}\left(\mathrm{b}_{1}\right)+\mathcal{G}\left(\mathrm{b}_{2}\right)\right]}{2^{s}} .
\end{aligned}
$$

Proof. Since $\mathcal{G}$ is $\varrho$-s-convex function, we have

$$
2^{s} \mathcal{G}\left(\varrho^{-1}\left(\frac{\varrho(x)+\varrho(y)}{2}\right)\right) \leq \mathcal{G}(x)+\mathcal{G}(y) .
$$


Putting,

$x=\varrho^{-1}\left(\frac{\kappa}{2} \varrho\left(\mathbf{b}_{1}\right)+\frac{2-\kappa}{2} \varrho\left(\mathbf{b}_{2}\right)\right)$ and

$y=\varrho^{-1}\left(\frac{2-\kappa}{2} \varrho\left(\mathrm{b}_{1}\right)+\frac{\kappa}{2} \varrho\left(\mathrm{b}_{1}\right)\right)$ in (21), multiplying by $\kappa^{\alpha-1}$ and then integrating over $[0,1]$, we have

$$
\begin{aligned}
& 2^{s} \int_{0}^{1} \mathcal{G}\left(\varrho^{-1}\left(\frac{\varrho\left(\mathrm{b}_{1}\right)+\varrho\left(\mathrm{b}_{2}\right)}{2}\right)\right) \kappa^{\alpha-1} d \kappa \\
& \leq \int_{0}^{1} \mathcal{G}\left(\varrho^{-1}\left(\frac{\kappa}{2} \varrho\left(\mathrm{b}_{1}\right)+\frac{2-\kappa}{2} \varrho\left(\mathrm{b}_{2}\right)\right)\right) \kappa^{\alpha-1} d \kappa \\
& \quad+\int_{0}^{1} \mathcal{G}\left(\varrho^{-1}\left(\frac{2-\kappa}{2} \varrho\left(\mathrm{b}_{1}\right)+\frac{\kappa}{2} \varrho\left(\mathrm{b}_{1}\right)\right)\right) \kappa^{\alpha-1} d \kappa
\end{aligned}
$$

By applying the change of variable technique, (22) becomes:

$$
\begin{aligned}
& u=\varrho^{-1}\left(\frac{\kappa}{2} \varrho\left(\mathbf{b}_{1}\right)+\frac{2-\kappa}{2} \varrho\left(\mathbf{b}_{2}\right)\right) \text { or } \kappa=\frac{2\left(\varrho(u)-\varrho\left(\mathbf{b}_{2}\right)\right)}{\varrho\left(\mathbf{b}_{1}\right)-\mathbf{b}_{2}} \text { and } d \kappa=\frac{2 \varrho^{\prime}(u) d u}{\varrho\left(\mathbf{b}_{1}\right)-\varrho\left(\mathbf{b}_{2}\right)} \\
& v=\varrho^{-1}\left(\frac{2-\kappa}{2} \varrho\left(\mathbf{b}_{1}\right)+\frac{\kappa}{2} \varrho\left(\mathbf{b}_{1}\right)\right) \text { or } \kappa=\frac{2\left(\varrho(v)-\varrho\left(\mathbf{b}_{1}\right)\right)}{\varrho\left(\mathbf{b}_{2}\right)-\mathrm{b}_{1}} \text { and } d \kappa=\frac{2 \varrho^{\prime}(v) d v}{\varrho\left(\mathbf{b}_{2}\right)-\varrho\left(\mathbf{b}_{1}\right)} \\
& \mathcal{G}\left(\varrho^{-1}\left(\frac{\varrho\left(\mathrm{b}_{1}\right)+\varrho\left(\mathrm{b}_{2}\right)}{2}\right)\right)
\end{aligned}
$$

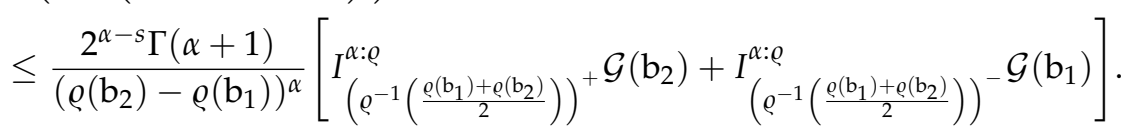

For the second inequality, we use the definition of $\varrho$-s convexity, i.e.,

$$
\begin{aligned}
& \mathcal{G}\left(\varrho^{-1}\left(\frac{\kappa}{2} \varrho\left(\mathrm{b}_{1}\right)+\frac{2-\kappa}{2} \varrho\left(\mathrm{b}_{2}\right)\right)\right) \leq\left(\frac{\kappa}{2}\right)^{S} \mathcal{G}\left(\mathrm{b}_{1}\right)+\left(\frac{2-\kappa}{2}\right)^{S} \mathcal{G}\left(\mathrm{b}_{2}\right) . \\
& \mathcal{G}\left(\varrho^{-1}\left(\frac{2-\kappa}{2} \varrho\left(\mathrm{b}_{1}\right)+\frac{\kappa}{2} \varrho\left(\mathrm{b}_{2}\right)\right)\right) \leq\left(\frac{2-\kappa}{2}\right)^{S} \mathcal{G}\left(\mathrm{b}_{1}\right)+\left(\frac{\kappa}{2}\right)^{S} \mathcal{G}\left(\mathrm{b}_{2}\right) .
\end{aligned}
$$

Upon adding both the inequalities, we have

$$
\begin{aligned}
\left.\mathcal{G}\left(\varrho^{-1}\left(\frac{\kappa}{2} \varrho\left(\mathrm{b}_{1}\right)+\frac{2-\kappa}{2} \varrho\left(\mathrm{b}_{2}\right)\right)\right)+\mathcal{G}\left(\varrho^{-1}\left(\frac{2-\kappa}{2} \varrho\left(\mathrm{b}_{1}\right)+\frac{\kappa}{2} \varrho\left(\mathrm{b}_{2}\right)\right)\right)\right) \\
\leq\left[\mathcal{G}\left(\mathrm{b}_{1}\right)+\mathcal{G}\left(\mathrm{b}_{2}\right)\right]\left[\frac{(2-\kappa)^{s}+\kappa^{\mathcal{S}}}{2^{s}}\right] .
\end{aligned}
$$

Multiplying by $\kappa^{\alpha-1}$ (24) and integrating with respect to $\kappa$ over $[0,1]$, we get

$$
\begin{aligned}
& \int_{0}^{1} \mathcal{G}\left(\varrho^{-1}\left(\frac{\kappa}{2} \varrho\left(\mathrm{b}_{1}\right)+\frac{2-\kappa}{2} \varrho\left(\mathrm{b}_{2}\right)\right)\right) \kappa^{\alpha-1} d \kappa \\
& \left.+\int_{0}^{1} \mathcal{G}\left(\varrho^{-1}\left(\frac{2-\kappa}{2} \varrho\left(\mathrm{b}_{1}\right)+\frac{\kappa}{2} \varrho\left(\mathrm{b}_{2}\right)\right)\right)\right) \kappa^{\alpha-1} d \kappa \\
& \leq\left[\mathcal{G}\left(\mathrm{b}_{1}\right)+\mathcal{G}\left(\mathrm{b}_{2}\right)\right] \int_{0}^{1}\left[\frac{(2-\kappa)^{S}+\kappa^{S}}{2^{s}}\right] \kappa^{\alpha-1} d \kappa .
\end{aligned}
$$

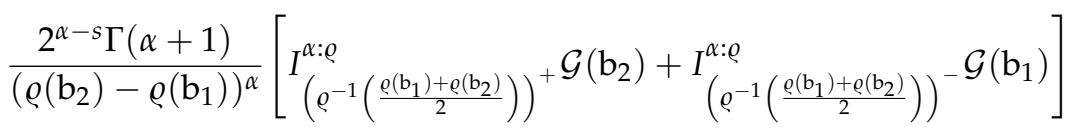

$$
\begin{aligned}
& \leq\left[\frac{1}{2^{s}(s+\alpha)}+2^{\alpha} \beta_{\frac{1}{2}}(\alpha, s+1)\right] \frac{\alpha\left[\mathcal{G}\left(\mathrm{b}_{1}\right)+\mathcal{G}\left(\mathrm{b}_{2}\right)\right]}{2^{s}} .
\end{aligned}
$$

The result is established by comparing (23) and (25) . 
Remark 8. Under the assumption of Theorem 9 and $s=1$

$$
\begin{aligned}
& \mathcal{G}\left(\varrho^{-1}\left(\frac{\varrho\left(\mathbf{b}_{1}\right)+\varrho\left(\mathbf{b}_{2}\right)}{2}\right)\right)
\end{aligned}
$$

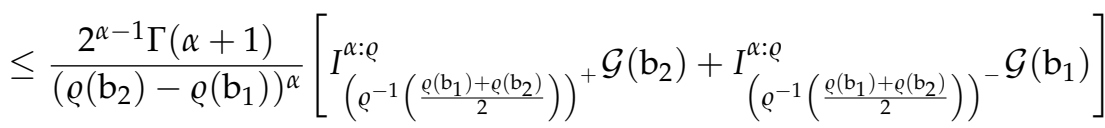

$$
\begin{aligned}
& \leq\left[\frac{1}{2(1+\alpha)}+2^{\alpha} \beta_{\frac{1}{2}}(\alpha, 2)\right] \frac{\alpha\left[\mathcal{G}\left(b_{1}\right)+\mathcal{G}\left(b_{2}\right)\right]}{2} .
\end{aligned}
$$

Remark 9. Under the assumption of Theorem 9 when $\varrho(x)=x$ and $\alpha=1$

$$
\begin{aligned}
2^{2 s} \mathcal{G}\left(\left(\frac{\mathrm{b}_{1}+\mathrm{b}_{2}}{2}\right)\right) \leq \frac{2^{s+2}}{\mathrm{~b}_{2}-\mathrm{b}_{1}}\left[I_{\left(\frac{\mathrm{b}_{1}+\mathrm{b}_{2}}{2}\right)^{+}} \mathcal{G}\left(\mathrm{b}_{2}\right)+I_{\left(\frac{\mathrm{b}_{1}+\mathrm{b}_{2}}{2}\right)^{-}} \mathcal{G}\left(\mathrm{b}_{1}\right)\right] \\
\leq 2^{1-2 s}\left[\frac{1}{2^{s}(s+1)}+2 \beta_{\frac{1}{2}}(1, s+1)\right]\left[\mathcal{G}\left(\mathrm{b}_{1}\right)+\mathcal{G}\left(\mathrm{b}_{2}\right)\right]
\end{aligned}
$$

Pachpatte-Type Fractional Inequalities

Theorem 10. Let $\mathcal{G}$ and $\mathcal{H}$ be integrable $\varrho$-s convex functions with respect to the function $\varrho$ on $\left[\mathrm{b}_{1}, \mathrm{~b}_{2}\right]$. Then the following fractional integral inequality holds:

$$
\begin{aligned}
& \frac{\Gamma(\alpha)}{\left(\varrho\left(\mathrm{b}_{2}\right)-\varrho\left(\mathrm{b}_{1}\right)\right)^{\alpha}}\left[I_{\mathrm{b}_{1}{ }^{+}}^{\alpha: \varrho} \mathcal{G}\left(\mathrm{b}_{2}\right) \mathcal{H}\left(\mathrm{b}_{2}\right)+I_{\mathrm{b}_{2}-\varrho}^{\alpha:} \mathcal{G}\left(\mathrm{b}_{1}\right) \mathcal{H}\left(\mathrm{b}_{1}\right)\right] \\
& \leq M\left(\mathrm{~b}_{1}, \mathrm{~b}_{2}\right)\left[\frac{1}{2 s+\alpha}+\beta(2 s+1, \alpha)\right]+2 N\left(\mathrm{~b}_{1}, \mathrm{~b}_{2}\right) \beta(s+1, s+\alpha)
\end{aligned}
$$

Proof. Using the definition of $\varrho$-s convex functions, we have

$$
\mathcal{G}\left(\varrho^{-1}\left(\kappa \varrho\left(b_{1}\right)+(1-\kappa) \varrho\left(b_{2}\right)\right)\right) \leq \kappa^{s} \mathcal{G}\left(b_{1}\right)+(1-\kappa)^{s} \mathcal{G}\left(b_{2}\right),
$$

and

$$
\mathcal{H}\left(\varrho^{-1}\left(\kappa \varrho\left(\mathrm{b}_{1}\right)+(1-\kappa) \varrho\left(\mathrm{b}_{2}\right)\right)\right) \leq \kappa^{s} \mathcal{H}\left(\mathrm{b}_{1}\right)+(1-\kappa)^{s} \mathcal{H}\left(\mathrm{b}_{2}\right) .
$$

Multiplying both the above equations side by side, we have

$$
\begin{aligned}
& \mathcal{G}\left(\varrho^{-1}\left(\kappa \varrho\left(\mathrm{b}_{1}\right)+(1-\kappa) \varrho\left(\mathrm{b}_{2}\right)\right)\right) \mathcal{H}\left(\varrho^{-1}\left(\kappa \varrho\left(\mathrm{b}_{1}\right)+(1-\kappa) \varrho\left(\mathrm{b}_{2}\right)\right)\right) \\
& \quad \leq \kappa^{2 s} \mathcal{G}\left(\mathrm{b}_{1}\right) \mathcal{H}\left(\mathrm{b}_{1}\right)+(1-\kappa)^{2 s} \mathcal{G}\left(\mathrm{b}_{2}\right) \mathcal{H}\left(\mathrm{b}_{2}\right)+(1-\kappa)^{s} \kappa^{s}\left[\mathcal{G}\left(\mathrm{b}_{1}\right) \mathcal{H}\left(\mathrm{b}_{2}\right)+\mathcal{G}\left(\mathrm{b}_{2}\right) \mathcal{H}\left(\mathrm{b}_{1}\right)\right]
\end{aligned}
$$

Consequently, we also have

$$
\begin{aligned}
& \mathcal{G}\left(\varrho^{-1}\left(\kappa \varrho\left(\mathrm{b}_{2}\right)+(1-\kappa) \varrho\left(\mathrm{b}_{1}\right)\right)\right) \mathcal{H}\left(\varrho^{-1}\left(\kappa \varrho\left(\mathrm{b}_{2}\right)+(1-\kappa) \varrho\left(\mathrm{b}_{1}\right)\right)\right) \\
& \quad \leq \kappa^{2 s} \mathcal{G}\left(\mathrm{b}_{2}\right) \mathcal{H}\left(\mathrm{b}_{2}\right)+(1-\kappa)^{2 s} \mathcal{G}\left(\mathrm{b}_{1}\right) \mathcal{H}\left(\mathrm{b}_{1}\right)+(1-\kappa)^{s} \kappa^{S}\left[\mathcal{G}\left(\mathrm{b}_{2}\right) \mathcal{H}\left(\mathrm{b}_{1}\right)+\mathcal{G}\left(\mathrm{b}_{1}\right) \mathcal{H}\left(\mathrm{b}_{2}\right)\right]
\end{aligned}
$$

Upon adding,

$$
\begin{aligned}
& \mathcal{G}\left(\varrho^{-1}\left(\kappa \varrho\left(\mathrm{b}_{1}\right)+(1-\kappa) \varrho\left(\mathrm{b}_{2}\right)\right)\right) \mathcal{H}\left(\varrho^{-1}\left(\kappa \varrho\left(\mathrm{b}_{1}\right)+(1-\kappa) \varrho\left(\mathrm{b}_{2}\right)\right)\right) \\
+ & \mathcal{G}\left(\varrho^{-1}\left(\kappa \varrho\left(\mathrm{b}_{2}\right)+(1-\kappa) \varrho\left(\mathrm{b}_{1}\right)\right)\right) \mathcal{H}\left(\varrho^{-1}\left(\kappa \varrho\left(\mathrm{b}_{2}\right)+(1-\kappa) \varrho\left(\mathrm{b}_{1}\right)\right)\right) \\
\leq & {\left[\kappa^{2 s}+(1-\kappa)^{2 s}\right]\left[\mathcal{G}\left(\mathrm{b}_{1}\right) \mathcal{H}\left(\mathrm{b}_{1}\right)+\mathcal{G}\left(\mathrm{b}_{2}\right) \mathcal{H}\left(\mathrm{b}_{2}\right)\right]+2 \kappa^{\mathcal{S}}(1-\kappa)^{S}\left[\mathcal{G}\left(\mathrm{b}_{1}\right) \mathcal{H}\left(\mathrm{b}_{2}\right)+\mathcal{G}\left(\mathrm{b}_{2}\right) \mathcal{H}\left(\mathrm{b}_{1}\right)\right] . }
\end{aligned}
$$


Multiplying both the sides by $\kappa^{\alpha-1}$ and then integrating with respect to $\kappa$,

$$
\begin{aligned}
& \int_{0}^{1} \kappa^{\alpha-1} \mathcal{G}\left(\varrho^{-1}\left(\kappa \varrho\left(\mathrm{b}_{1}\right)+(1-\kappa) \varrho\left(\mathrm{b}_{2}\right)\right)\right) \mathcal{H}\left(\varrho^{-1}\left(\kappa \varrho\left(\mathrm{b}_{1}\right)+(1-\kappa) \varrho\left(\mathrm{b}_{2}\right)\right)\right) d \kappa \\
+ & \int_{0}^{1} \kappa^{\alpha-1} \mathcal{G}\left(\varrho^{-1}\left(\kappa \varrho\left(\mathrm{b}_{2}\right)+(1-\kappa) \varrho\left(\mathrm{b}_{1}\right)\right)\right) \mathcal{H}\left(\varrho^{-1}\left(\kappa \varrho\left(\mathrm{b}_{2}\right)+(1-\kappa) \varrho\left(\mathrm{b}_{1}\right)\right)\right) d \kappa \\
\leq & {\left[\mathcal{G}\left(\mathrm{b}_{1}\right) \mathcal{H}\left(\mathrm{b}_{1}\right)+\mathcal{G}\left(\mathrm{b}_{2}\right) \mathcal{H}\left(\mathrm{b}_{2}\right)\right] \int_{0}^{1} \kappa^{\alpha-1}\left[\kappa^{2 s}+(1-\kappa)^{2 s}\right] d \kappa } \\
& +2\left[\mathcal{G}\left(\mathrm{b}_{1}\right) \mathcal{H}\left(\mathrm{b}_{2}\right)+\mathcal{G}\left(\mathrm{b}_{2}\right) \mathcal{H}\left(\mathrm{b}_{1}\right)\right] \int_{0}^{1} \kappa^{\alpha-1} \kappa^{s}(1-\kappa)^{s} d \kappa .
\end{aligned}
$$

Consequently,

$$
\begin{aligned}
& \frac{1}{\left(\varrho\left(\mathrm{b}_{2}\right)-\varrho\left(\mathrm{b}_{1}\right)\right)^{\alpha}} \int_{\mathrm{b}_{1}}^{\mathrm{b}_{2}} \mathcal{G}(x) \mathcal{H}(x) \varrho^{\prime}(x)\left(\varrho\left(\mathrm{b}_{2}\right)-\varrho\left(\mathrm{b}_{1}\right)\right)^{\alpha-1} d x \\
& +\frac{1}{\left(\varrho\left(\mathrm{b}_{2}\right)-\varrho\left(\mathrm{b}_{1}\right)\right)^{\alpha}} \int_{\mathrm{b}_{1}}^{\mathrm{b}_{2}} \mathcal{G}(y) \mathcal{H}(y) \varrho^{\prime}(y)\left(\varrho\left(\mathrm{b}_{2}\right)-\varrho\left(\mathrm{b}_{1}\right)\right)^{\alpha-1} d y \\
& \leq M\left(\mathrm{~b}_{1}, \mathrm{~b}_{2}\right)\left[\frac{1}{2 s+\alpha}+\beta(2 s+1, \alpha)\right]+2 N\left(\mathrm{~b}_{1}, \mathrm{~b}_{2}\right) \beta(s+1, s+\alpha) .
\end{aligned}
$$

Using the definition of the $\Psi$ - Riemann-Liouville fractional operator, we have

$$
\begin{aligned}
& \frac{\Gamma(\alpha)}{(\varrho(b)-\varrho(a))^{\alpha}}\left[I_{\mathrm{b}_{1}+}^{\alpha: \varrho} \mathcal{G}\left(\mathrm{b}_{2}\right) \mathcal{H}\left(\mathrm{b}_{2}\right)+I_{\mathrm{b}_{2}-}^{\alpha: \varrho} \mathcal{G}\left(\mathrm{b}_{1}\right) \mathcal{H}\left(\mathrm{b}_{1}\right)\right] \\
& \leq M\left(\mathrm{~b}_{1}, \mathrm{~b}_{2}\right)\left[\frac{1}{2 s+\alpha}+\beta(2 s+1, \alpha)\right]+2 N\left(\mathrm{~b}_{1}, \mathrm{~b}_{2}\right) \beta(s+1, s+\alpha) .
\end{aligned}
$$

This completes the proof.

Theorem 11. Let $\mathcal{G}$ and $\mathcal{H}$ be integrable $\varrho$-s convex functions with respect to the function $\varrho$ on $\left[\mathrm{b}_{1}, \mathrm{~b}_{2}\right]$. Then, the following fractional integral inequality holds:

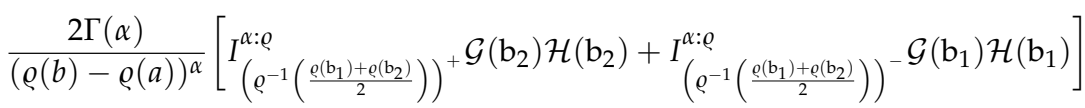

$$
\begin{aligned}
& \leq M\left(\mathrm{~b}_{1}, \mathrm{~b}_{2}\right)\left[\left[\frac{1}{4^{s}(2 s+\alpha)}\right]+\frac{{ }_{2} F_{1}(\alpha,-2 s, \alpha+1,1 / 2)}{\alpha}\right]+N\left(\mathrm{~b}_{1}, \mathrm{~b}_{2}\right)\left[2^{\alpha} \beta(1 / 2, \alpha+s), s+1\right] .
\end{aligned}
$$

Proof. From the definition of $\varrho$-s convex function, we have

$$
\begin{aligned}
& \mathcal{G}\left(\varrho^{-1}\left(\frac{\kappa}{2} \varrho\left(\left(\mathrm{b}_{1}\right)\right)+\frac{2-\kappa}{2} \varrho\left(\mathrm{b}_{2}\right)\right)\right) \leq\left(\frac{\kappa}{2}\right)^{s} \mathcal{G}\left(\mathrm{b}_{1}\right)+\left(\frac{2-\kappa}{2}\right)^{s} \mathcal{G}\left(\mathrm{b}_{2}\right) . \\
& \mathcal{H}\left(\varrho^{-1}\left(\frac{\kappa}{2} \varrho\left(\mathrm{b}_{1}\right)+\frac{2-\kappa}{2} \varrho\left(\mathrm{b}_{2}\right)\right)\right) \leq\left(\frac{t}{2}\right)^{s} \mathcal{H}\left(\mathrm{b}_{1}\right)+\left(\frac{2-\kappa}{2}\right)^{s} \mathcal{H}\left(\mathrm{b}_{2}\right) .
\end{aligned}
$$

Multiplying both the inequalities side by side, we have

$$
\begin{aligned}
& \mathcal{G}\left(\varrho^{-1}\left(\frac{\kappa}{2} \varrho\left(\mathrm{b}_{1}\right)+\frac{2-\kappa}{2} \varrho\left(\mathrm{b}_{2}\right)\right)\right) \mathcal{H}\left(\varrho^{-1}\left(\frac{\kappa}{2} \varrho\left(\mathrm{b}_{1}\right)+\frac{2-\kappa}{2} \varrho\left(\mathrm{b}_{2}\right)\right)\right) \\
& \leq\left(\frac{\kappa}{2}\right)^{2 s} \mathcal{G}\left(\mathrm{b}_{1}\right) \mathcal{H}\left(\mathrm{b}_{1}\right)+\left(\frac{2-\kappa}{2}\right)^{s} \mathcal{G}\left(\mathrm{b}_{2}\right) \mathcal{H}\left(\mathrm{b}_{2}\right) \\
& +\left(\frac{\kappa}{2}\right)^{s} \mathcal{H}\left(\mathrm{b}_{2}\right)\left(\frac{2-\kappa}{2}\right)^{s}\left[\mathcal{G}\left(\mathrm{b}_{1}\right) \mathcal{H}\left(\mathrm{b}_{2}\right)+\mathcal{G}\left(\mathrm{b}_{2}\right) \mathcal{H}\left(\mathrm{b}_{1}\right)\right] .
\end{aligned}
$$


Similarly,

$$
\begin{aligned}
& \mathcal{G}\left(\varrho^{-1}\left(\frac{\kappa}{2} \varrho\left(\mathrm{b}_{2}\right)+\frac{2-\kappa}{2} \varrho\left(\mathrm{b}_{1}\right)\right)\right) \mathcal{H}\left(\varrho^{-1}\left(\frac{\kappa}{2} \varrho\left(\mathrm{b}_{2}\right)+\frac{2-\kappa}{2} \varrho\left(\mathrm{b}_{1}\right)\right)\right) \\
& \leq\left(\frac{t}{2}\right)^{2 s} \mathcal{G}\left(\mathrm{b}_{2}\right) \mathcal{H}\left(\mathrm{b}_{2}\right)+\left(\frac{2-\kappa}{2}\right)^{s} \mathcal{G}\left(\mathrm{b}_{1}\right) \mathcal{H}\left(\mathrm{b}_{1}\right) \\
& +\left(\frac{\kappa}{2}\right)^{S} \mathcal{H}\left(\mathrm{b}_{2}\right)\left(\frac{2-\kappa}{2}\right)^{s}\left[\mathcal{G}\left(\mathrm{b}_{1}\right) \mathcal{H}\left(\mathrm{b}_{2}\right)+\mathcal{G}\left(\mathrm{b}_{2}\right) \mathcal{H}\left(\mathrm{b}_{1}\right)\right] .
\end{aligned}
$$

Upon adding,

$$
\begin{aligned}
& \mathcal{G}\left(\varrho^{-1}\left(\frac{\kappa}{2} \varrho\left(\mathrm{b}_{1}\right)+\frac{2-\kappa}{2} \varrho\left(\mathrm{b}_{2}\right)\right)\right) \mathcal{H}\left(\varrho^{-1}\left(\frac{\kappa}{2} \varrho\left(\mathrm{b}_{1}\right)+\frac{2-\kappa}{2} \varrho\left(\mathrm{b}_{2}\right)\right)\right) \\
& +\mathcal{G}\left(\varrho^{-1}\left(\frac{\kappa}{2} \varrho\left(\mathrm{b}_{2}\right)+\frac{2-\kappa}{2} \varrho\left(\mathrm{b}_{1}\right)\right)\right) \mathcal{H}\left(\varrho^{-1}\left(\frac{\kappa}{2} \varrho\left(\mathrm{b}_{2}\right)+\frac{2-\kappa}{2} \varrho\left(\mathrm{b}_{1}\right)\right)\right) \\
& \leq\left(\frac{\kappa}{2}\right)^{2 s}\left[\mathcal{G}\left(\mathrm{b}_{1}\right) \mathcal{H}\left(\mathrm{b}_{1}\right)+\mathcal{G}\left(\mathrm{b}_{2}\right) \mathcal{H}\left(\mathrm{b}_{2}\right)\right]+\left(\frac{2-\kappa}{2}\right)^{2 s}\left[\mathcal{G}\left(\mathrm{b}_{1}\right)+\mathcal{H}\left(\mathrm{b}_{1}\right)+\mathcal{G}\left(\mathrm{b}_{2}\right) \mathcal{H}\left(\mathrm{b}_{2}\right)\right] \\
& +2\left(\frac{\kappa}{2}\right)^{s}\left(\frac{2-\kappa}{2}\right)^{s}\left[\mathcal{G}\left(\mathrm{b}_{1}\right) \mathcal{H}\left(\mathrm{b}_{2}\right)+\mathcal{G}\left(\mathrm{b}_{2}\right) \mathcal{H}\left(\mathrm{b}_{1}\right)\right] \\
& =\left[\left(\frac{\kappa}{2}\right)^{2 s}+\left(\frac{2-\kappa}{2}\right)^{2 s}\right] M\left(\mathrm{~b}_{1}, \mathrm{~b}_{2}\right)+\left(\frac{\kappa}{2}\right)^{2 s}\left(\frac{2-\kappa}{2}\right)^{2 s} N\left(\mathrm{~b}_{1}, \mathrm{~b}_{2}\right) .
\end{aligned}
$$

Upon multipling both sides of the above inequality by $\kappa^{\alpha-1}$ and then integrating with respect to $\kappa$ over $[0,1]$, we get

$$
\text { Let } \begin{aligned}
\varrho^{-1}\left(\frac{\kappa}{2} \varrho\left(\mathrm{b}_{1}\right)+\frac{2-\kappa}{2} \varrho\left(\mathrm{b}_{2}\right)\right)=\mathrm{u} \\
\quad \Longrightarrow \frac{\kappa}{2} \varrho\left(\mathrm{b}_{1}\right)+\frac{2-\kappa}{2} \varrho\left(\mathrm{b}_{2}\right)=\varrho(\mathrm{u}) \\
\Longrightarrow \kappa=\frac{2\left(\varrho(\mathrm{u})-\varrho\left(\mathrm{b}_{2}\right)\right)}{\varrho\left(\mathrm{b}_{1}\right)-\varrho\left(\mathrm{b}_{2}\right)}=\frac{2\left(\varrho\left(\mathrm{b}_{2}\right)-\varrho(\mathrm{u})\right)}{\varrho\left(\mathrm{b}_{2}\right)-\varrho\left(\mathrm{b}_{1}\right)} \\
\Longrightarrow d \kappa=\frac{-2 \varrho^{\prime}(\mathrm{u})}{\varrho\left(\mathrm{b}_{2}\right)-\varrho\left(\mathrm{b}_{1}\right)} d \mathrm{u} .
\end{aligned}
$$

This implies

$$
\begin{aligned}
& \int_{0}^{1} \kappa^{\alpha-1} \mathcal{G}\left(\varrho^{-1}\left(\frac{\kappa}{2} \varrho\left(\mathrm{b}_{1}\right)+\frac{2-\kappa}{2} \varrho\left(\mathrm{b}_{2}\right)\right)\right) \mathcal{H}\left(\varrho^{-1}\left(\frac{\kappa}{2} \varrho\left(\mathrm{b}_{1}\right)+\frac{2-\kappa}{2} \varrho\left(\mathrm{b}_{2}\right)\right)\right) d \kappa \\
= & \int_{b}^{\varrho^{-1}\left(\frac{\rho\left(\mathrm{b}_{1}\right)+\rho\left(\mathrm{b}_{2}\right)}{2}\right)} \mathcal{G}(\mathrm{u}) \mathcal{H}(\mathrm{u})\left(\frac{2\left(\varrho\left(\mathrm{b}_{2}\right)-\varrho(\mathrm{u})\right)}{\varrho\left(\mathrm{b}_{2}\right)-\varrho\left(\mathrm{b}_{1}\right)}\right)^{\alpha-1} \frac{-2 \varrho^{\prime}(\mathrm{u})}{\varrho\left(\mathrm{b}_{2}\right)-\varrho\left(\mathrm{b}_{1}\right)} d \mathrm{u} \\
= & \frac{2 \Gamma(\alpha)}{(\varrho(b)-\varrho(a))^{\alpha}} I^{\alpha: \varrho}\left(\varrho^{-1}\left(\frac{\rho\left(\mathrm{b}_{1}\right)+\varrho\left(\mathrm{b}_{2}\right)}{2}\right)\right)^{+} \mathcal{G}\left(\mathrm{b}_{2}\right) \mathcal{H}\left(\mathrm{b}_{2}\right) .
\end{aligned}
$$

Similarly,

$$
\begin{aligned}
& \int_{0}^{1} \kappa^{\alpha-1} \mathcal{G}\left(\varrho^{-1}\left(\frac{\kappa}{2} \varrho\left(\mathrm{b}_{2}\right)+\frac{2-\kappa}{2} \varrho\left(\mathrm{b}_{1}\right)\right)\right) \mathcal{H}\left(\varrho^{-1}\left(\frac{\kappa}{2} \varrho\left(\mathrm{b}_{2}\right)+\frac{2-\kappa}{2} \varrho\left(\mathrm{b}_{1}\right)\right)\right) d \kappa \\
= & \frac{2 \Gamma(\alpha)}{(\varrho(b)-\varrho(a))^{\alpha}} I^{\alpha \cdot \varrho}\left(\varrho^{-1}\left(\frac{\varrho\left(\mathrm{b}_{1}\right)+\varrho\left(\mathrm{b}_{2}\right)}{2}\right)\right)^{-} \mathcal{G}\left(\mathrm{b}_{1}\right) \mathcal{H}\left(\mathrm{b}_{1}\right) .
\end{aligned}
$$


Also,

$$
\begin{aligned}
& M\left(\mathrm{~b}_{1}, \mathrm{~b}_{2}\right) \int_{0}^{1}\left[\left(\frac{\kappa}{2}\right)^{2 s}+\left(\frac{2-\kappa}{2}\right)^{2 s}\right] d \kappa+N\left(\mathrm{~b}_{1}, \mathrm{~b}_{2}\right) \int_{0}^{1}\left(\frac{\kappa}{2}\right)^{2 s}\left(\frac{2-\kappa}{2}\right)^{2 s} d \kappa \\
= & M\left(\mathrm{~b}_{1}, \mathrm{~b}_{2}\right)\left[\left[\frac{1}{4^{s}(2 s+\alpha)}\right]+\frac{{ }_{2} F_{1}(\alpha,-2 s, \alpha+1,1 / 2)}{\alpha}\right]+N\left(\mathrm{~b}_{1}, \mathrm{~b}_{2}\right)\left[2^{\alpha} \beta(1 / 2, \alpha+s, s+1)\right] .
\end{aligned}
$$

By arranging the above computations, we have the desired proof.

\section{Applications}

\subsection{Special Means}

Now, we propose some applications to special means of real numbers related to our established results.

1. The arithmetic mean:

$$
\mathcal{A}=\mathcal{A}\left(\mathrm{b}_{1}, \mathrm{~b}_{2}\right)=\frac{\mathrm{b}_{1}+\mathrm{b}_{2}}{2}, \mathrm{~b}_{1}, \mathrm{~b}_{2} \in \mathcal{R}
$$

2. The geometric mean:

$$
\mathcal{G}=\mathcal{G}\left(\mathrm{b}_{1}, \mathrm{~b}_{2}\right)=\sqrt{\mathrm{b}_{1} \mathrm{~b}_{2}}
$$

3. The logarithmic mean:

$$
\mathcal{L}\left(b_{1}, b_{2}\right)=\frac{b_{2}-b_{1}}{\ln \left|b_{2}\right|-\ln \left|b_{1}\right|}, \quad b_{1} \neq b_{2}, b_{1} b_{2} \neq 0 .
$$

Proposition 1. Let $\mathrm{b}_{1}, \mathrm{~b}_{2} \in \mathcal{R}^{+}, \mathrm{b}_{1}<\mathrm{b}_{2}$; then

$$
\left[e^{\mathcal{A}\left(\ln \mathrm{b}_{1}, \ln _{2}\right)}\right]^{s} \leq \frac{1}{2^{s-1}\left(\ln \mathrm{b}_{2}-\ln \mathrm{b}_{1}\right)}\left[\frac{\mathrm{b}_{2}{ }^{s}-\mathrm{b}_{1}{ }^{s}}{s}\right] \leq\left[\frac{\mathcal{A}\left(\mathrm{b}_{1}{ }^{s}, \mathrm{~b}_{2}{ }^{s}\right)}{2^{s-2}[s+1]}\right] .
$$

Proof. In Theorem 5, setting $\mathcal{G}(x)=x^{\mathcal{S}}$ with $\varrho(x)=\ln x$ completes the proof.

Proposition 2. Let $\mathrm{b}_{1}, \mathrm{~b}_{2} \in \mathcal{R}^{+}, \mathrm{b}_{1}<\mathrm{b}_{2}$; then

$$
\frac{1}{e^{\mathcal{A}\left(\ln \mathrm{b}_{1}, \ln \mathrm{b}_{2}\right)}} \leq \frac{1}{2^{s-1}}\left[\frac{\mathcal{L}\left(\mathrm{b}_{2}, \mathrm{~b}_{1}\right)}{\mathcal{G}^{2}\left(\mathrm{~b}_{1}, \mathrm{~b}_{2}\right)}\right] \leq \frac{1}{2^{s-2}[s+1]}\left[\frac{\mathcal{A}\left(\mathrm{b}_{2}, \mathrm{~b}_{1}\right)}{\mathcal{G}^{2}\left(\mathrm{~b}_{1}, \mathrm{~b}_{2}\right)}\right] .
$$

Proof. In Theorem 5, setting $\mathcal{G}(x)=\frac{1}{x}$ with $\varrho(x)=\ln x$ completes the proof.

6.2. q-Digamma Function

Example 1. The $q$-digamma(psi) function $\varrho_{\rho}$ is the $\rho$-analogue of the digamma function $\varrho$ (see [54]) given as:

$$
\begin{aligned}
\varrho_{\rho} & =-\ln (1-\rho)+\ln \rho \sum_{k=0}^{\infty} \frac{\rho^{k+\gamma}}{1-\rho^{k+\gamma}} \\
& =-\ln (1-\rho)+\ln \rho \sum_{k=0}^{\infty} \frac{\rho^{k \gamma}}{1-\rho^{k \gamma}} .
\end{aligned}
$$

For $\rho>1$ and $\gamma>0, \rho$-digamma function $\varrho_{\rho}$ can be given as: 


$$
\begin{aligned}
\varrho_{\rho} & =-\ln (\rho-1)+\ln \rho\left[\gamma-\frac{1}{2}-\sum_{k=0}^{\infty} \frac{\rho^{-(k+\gamma)}}{1-\rho^{-(k+\gamma)}}\right] \\
& =-\ln (\rho-1)+\ln \rho\left[\gamma-\frac{1}{2}-\sum_{k=0}^{\infty} \frac{\rho^{-k \gamma}}{1-\rho^{-k \gamma}}\right] .
\end{aligned}
$$

If we set $\mathcal{G}(x)=\psi_{\varrho}^{\prime}(x)$ in Remark 7 , then we have the following inequality.

$$
2^{s-1} \psi_{\varrho}^{\prime}\left(\frac{\mathrm{b}_{1}+\mathrm{b}_{2}}{2}\right) \leq \frac{\psi_{\varrho}\left(\mathrm{b}_{2}\right)-\psi_{\varrho}\left(\mathrm{b}_{1}\right)}{\mathrm{b}_{2}-\mathrm{b}_{1}} \leq \frac{\psi_{\varrho}^{\prime}\left(\mathrm{b}_{1}\right)+\psi_{\varrho}^{\prime}\left(\mathrm{b}_{2}\right)}{2(\varrho+1)(s+1)} .
$$

\subsection{Modified Bessel Functions}

Example 2. Let the function $\mathcal{J}_{\varrho}: \mathrm{R} \rightarrow[1, \infty)$ be defined [54] as

$$
\mathcal{J}_{\varrho}(u)=2^{\varrho} \Gamma(\varrho+1) u^{-\delta} \mathrm{I}_{\varrho}(u), \quad u \in \mathrm{R}
$$

Here, we consider the modified bessel function of first kind given in

$$
\mathrm{I}_{\varrho}(u)=\sum_{n=0}^{\infty} \frac{\left(\frac{u}{2}\right)^{\varrho+2 n}}{n ! \Gamma(\varrho+n+1)} .
$$

The first and second order derivative are given as

$$
\begin{gathered}
\mathcal{J}_{\varrho}^{\prime}(u)=\frac{u}{2(\varrho+1)} \mathcal{J}_{\varrho+1}(u) \\
\mathcal{J}_{\varrho}^{\prime \prime}(u)=\frac{1}{4(\varrho+1)}\left[\frac{u^{2}}{(\varrho+1)} \mathcal{J}_{\varrho+2}(u)+2 \mathcal{J}_{\varrho+1}(u)\right]
\end{gathered}
$$

If we use, $\Phi(u)=\mathcal{J}_{\varrho}^{\prime}(u)$ and the above functions in Remark 7, we have

$$
2^{s-3}\left(\frac{b_{1}+b_{2}}{\varrho+1}\right) \mathcal{J}_{\varrho+1}\left(\frac{b_{1}+b_{2}}{2}\right) \leq \frac{\left[\mathcal{J}_{\varrho}\left(b_{2}\right)-\mathcal{J}_{\varrho}\left(b_{1}\right)\right]}{b_{2}-b_{1}} \leq \frac{\left[b_{1} \mathcal{J}_{\varrho}\left(b_{1}\right)+b_{2} \mathcal{J}_{\varrho}\left(b_{2}\right)\right]}{2(\varrho+1)(s+1)} .
$$

\subsection{Matrices}

Here, we present some examples related to special functions and matrices.

Example 3. We represent $\mathbb{C}^{n}$ as the set of $n \times n$ complex matrices, $\mathbb{M}_{n}$ as the algebra of $n \times n$ complex matrices, and $\mathbb{M}_{n}^{+}$as the strictly positive matrices in $\mathbb{M}$. That is, $\mathrm{A} \in \mathbb{M}_{n}^{+}$, if $\langle\mathrm{A} u, u\rangle>0$ for all non-zero $u \in \mathbb{C}^{n}$.

Sababheh [55] proved that $\mathcal{G}(\kappa)=\left\|\mathrm{A}^{\kappa} \mathrm{XB}^{1-\kappa}+\mathrm{A}^{1-\kappa} \mathrm{XB}^{\kappa}\right\|, \mathrm{A}, \mathrm{B} \in \mathbb{M}_{n}^{+}, \mathrm{X} \in \mathbb{M}_{n}$ is convex for all $\kappa \in[0,1]$. We also have that every non-negative convex function is an s-convex function. Then, by using Theorem 8 for $\varrho(x)=x$, we have

$$
\begin{aligned}
& \left\|\mathrm{A}^{\left(\frac{\mathrm{b}_{1}+\mathrm{b}_{2}}{2}\right)} \mathrm{XB}^{1-\left(\frac{\mathrm{b}_{1}+\mathrm{b}_{2}}{2}\right)}+\mathrm{A}^{1-\left(\frac{\mathrm{b}_{1}+\mathrm{b}_{2}}{2}\right)} \mathrm{XB}^{\left(\frac{\mathrm{b}_{1}+\mathrm{b}_{2}}{2}\right)}\right\| \\
& \leq \frac{\Gamma(\alpha+1)}{2^{s}\left(\mathrm{~b}_{2}-\mathrm{b}_{1}\right)^{\alpha}} \\
& \times\left[\mathrm{I}_{\mathrm{b}_{1}{ }^{\alpha},}\left\|^{+} \mathrm{A}^{\mathrm{b}_{2}} \mathrm{XB}^{1-\mathrm{b}_{2}}+\mathrm{A}^{1-\mathrm{b}_{2}} \mathrm{XB}^{\mathrm{b}_{2}}\right\|+\mathrm{I}_{\mathrm{b}_{2}-}^{\alpha, \rho}\left\|\mathrm{A}^{\mathrm{b}_{1}} \mathrm{XB}^{1-\mathrm{b}_{1}}+\mathrm{A}^{1-\mathrm{b}_{1}} \mathrm{XB}^{\mathrm{b}_{1}}\right\|\right] \\
& \leq \frac{\alpha\left[\left\|\mathrm{A}^{\mathrm{b}_{1}} \mathrm{XB}^{1-\mathrm{b}_{1}}+\mathrm{A}^{1-\mathrm{b}_{1}} \mathrm{XB}^{\mathrm{b}_{1}}\right\|+\left\|\mathrm{A}^{\mathrm{b}_{2}} \mathrm{XB}^{1-\mathrm{b}_{2}}+\mathrm{A}^{1-\mathrm{b}_{2}} \mathrm{XB}^{\mathrm{b}_{2}}\right\|\right]}{2^{s}}\left[\frac{1}{s+\alpha}+\beta(s+1, \alpha)\right] .
\end{aligned}
$$

Furthermore, using Theorem 9 for $\varrho(x)=x$, we get 


$$
\begin{aligned}
& \left\|\mathrm{A}^{\left(\frac{\mathrm{b}_{1}+\mathrm{b}_{2}}{2}\right)} \mathrm{XB}^{1-\left(\frac{\mathrm{b}_{1}+\mathrm{b}_{2}}{2}\right)}+\mathrm{A}^{1-\left(\frac{\mathrm{b}_{1}+\mathrm{b}_{2}}{2}\right)} \mathrm{XB}^{\left(\frac{\mathrm{b}_{1}+\mathrm{b}_{2}}{2}\right)}\right\| \\
& \leq \frac{2^{\alpha-s} \Gamma(\alpha+1)}{\left(\mathrm{b}_{2}-\mathrm{b}_{1}\right)^{\alpha}}\left[\mathrm{I}^{\alpha}\left(\frac{\mathrm{b}_{1}+\mathrm{b}_{2}}{2}\right)^{+}+\left\|\mathrm{A}^{\mathrm{b}_{2}} \mathrm{XB}^{1-\mathrm{b}_{2}}+\mathrm{A}^{1-\mathrm{b}_{2}} \mathrm{XB}^{\mathrm{b}_{2}}\right\|\right. \\
& \left.+\mathrm{I}^{\alpha}\left(\frac{\mathrm{b}_{1}+\mathrm{b}_{2}}{2}\right)^{-}\left\|\mathrm{A}^{\mathrm{b}_{1}} \mathrm{XB}^{1-\mathrm{b}_{1}}+\mathrm{A}^{1-\mathrm{b}_{1}} \mathrm{XB}^{\mathrm{b}_{1}}\right\|\right] \\
& \leq \frac{\alpha\left[\left\|\mathrm{A}^{\mathrm{b}_{1}} \mathrm{XB}^{1-\mathrm{b}_{1}}+\mathrm{A}^{1-\mathrm{b}_{1}} \mathrm{XB}^{\mathrm{b}_{1}}\right\|+\left\|\mathrm{A}^{\mathrm{b}_{2}} \mathrm{XB}^{1-\mathrm{b}_{2}}+\mathrm{A}^{1-\mathrm{b}_{2}} \mathrm{XB}^{\mathrm{b}_{2}}\right\|\right]}{2^{s}} \\
& \times\left[\frac{1}{2^{s}(s+\alpha)}+2^{\alpha} \beta_{\frac{1}{2}}(s+1, \alpha)\right] .
\end{aligned}
$$

\section{Conclusions}

We have presented the ideas of $\varrho$-s-convex sets and $\varrho$-s-convex functions. Additionally, we have shown that this class of convexity incorporates a few different classes of traditional convexity. The $\varrho$-s-convex function is a speculation of convex functions identified with different power means. Moreover, by utilising the idea of $\varrho$-s-convexity, we have presented some new classical as well as fractional integral inequalities of the Hermite-Hadamard type and the Pachpatte type. The outcomes acquired in this article can help establish other kinds of inequalities. Additionally, these outcomes are general and can be determined to give further, possibly helpful, and intriguing inequalities via different types of fractional operators. In the future, our focus will be to incorporate these new class of convexities with concepts such as interval-valued calculus and fractional calculus. We anticipate that the thoughts and strategies in the paper might stimulate further investigations into this field.

Author Contributions: Conceptualization, S.K.S. and B.K.; methodology, S.K.S., M.T., H.A. and B.K.; software, S.K.S. and M.T. ; validation, H.A., B.K. and A.A.S.; formal analysis, H.A., B.K., A.A.S., T.B. and M.A.E.-S.; investigation, S.K.S. and M.T.; resources, S.K.S., M.T., H.A. and B.K.; writing-original draft preparation, S.K.S., M.T. and B.K.; writing-review and editing, S.K.S and M.T.; visualization, H.A., B.K., A.A.S., T.B. and M.A.E.-S.; supervision, H.A., B.K. and A.A.S.; project administration, H.A., B.K. and T.B.; funding acquisition, T.B. and M.A.E.-S. All authors have read and agreed to the published version of the manuscript.

Funding: This research received no external funding.

Institutional Review Board Statement: Not applicable.

Informed Consent Statement: Not applicable.

Data Availability Statement: Not applicable.

Conflicts of Interest: The authors declare no conflict of interest.

\section{List of Abbreviations}

The following abbreviations are used in this manuscript:

$\mathrm{H}-\mathrm{H} \quad$ Hermite-Hadamard

R-L Riemann-Liouville

\section{References}

1. Bertsimas, D.; Popescu, I. Optimal inequalities in probability theory: A convex optimization approach. SIAM J. Optim. 2005, 15, 780-804.

2. Lin, Z.; Bai, Z. Probability Inequalities of Random Variables. In Probability Inequalities; Springer: Berlin/Heidelberg, Germany, 2010; pp. 37-50.

3. Rumin, M. Spectral density and Sobolev inequalities for pure and mixed states. Geom. Funct. Anal. 2010, 20, 817-844. [CrossRef]

4. Sarikaya, M.Z.; Set, E.; Yaldiz, H.; Başak, N. Hermite-Hadamard's inequalities for fractional integrals and related fractional inequalities. Math. Comput. Model. 2013, 57, 2403-2407. [CrossRef] 
5. Bermudo, S.; Kórus, P.; Valdés, J.N. On q-Hermite-Hadamard inequalities for general convex functions. Acta Math. Hung. 2020, 162, 364-374. [CrossRef]

6. Zhao, D.; An, T.; Ye, G.; Liu, W. New Jensen and Hermite-Hadamard type inequalities for h-convex interval-valued functions. J Inequal. App. 2018, 2018, 302. [CrossRef]

7. Kotrys, D. Hermite-Hadamard inequality for convex stochastic processes. Aequationes Math. 2012, 83, 143-151. [CrossRef]

8. Rashid, S.; Noor, M.A.; Noor, K.I.; Safdar, F.; Chu, Y.M. Hermite-Hadamard type inequalities for the class of convex functions on time scale. Mathematics 2019, 7, 956. [CrossRef]

9. Almutairi, O.; Kılıçman, A. Generalized Integral Inequalities for Hermite-Hadamard-Type Inequalities via s-Convexity on Fractal Sets. Mathematics 2019, 7, 1065. [CrossRef]

10. Niculescu, C.P.; Persson, L.E. Convex Functions and Their Applications; Springer: New York, NY, USA, 2006.

11. Krishna, V.; Maenner, E. Convex potentials with an application to mechanism design. Econometrica 2001, 69, 1113-1119. [CrossRef]

12. Okubo, S.; Isihara, A. Inequality for convex functions in quantum-statistical mechanics. Physica 1972, 59, 228-240. [CrossRef]

13. Peajcariaac, J.E.; Tong, Y.L. Convex Functions, Partial Orderings, and Statistical Applications; Academic Press: Cambridge, MA, USA, 1992.

14. Murota, K.; Tamura, A. New characterizations of M-convex functions and their applications to economic equilibrium models with indivisibilities. Discret. Appl. Math. 2003, 131, 495-512. [CrossRef]

15. Kaijser, S.; Nikolova, L.; Persson, L.E.; Wedestig, A. Hardy type inequalities via convexity. Math. Inequal. Appl. $2005,8,403-417$. [CrossRef]

16. Gunawan, H.; Eridani. Fractional integrals and generalized Olsen inequalities. Kyungpook Math. J. 2009, 49, 31-39. [CrossRef]

17. Sawano, Y.; Wadade, H. On the Gagliardo-Nirenberg type inequality in the critical Sobolev-Morrey space. J. Fourier Anal. Appl. 2013, 19, 20-47. [CrossRef]

18. Kunt, M.; Iscan, I. Hermite-Hadamard-Fejér type inequalities for p-convex functions. Arab J. Math. Sci. 2017, 23, 215-230. [CrossRef]

19. Srivastava, H.M.; Tseng, K.-L.; Tseng, S.-J.; Lo, J.-C. Some weighted Opial-type inequalities on time scales. Taiwan J. Math. 2010, 14, 107-122. [CrossRef]

20. Luo, C.Y.; Du, T.S.; Kunt, M.; Zhang, Y. Certain new bounds considering the weighted Simpson-like type inequality and applications. J. Inequal. Appl. 2018, 2018, 332. [CrossRef]

21. Gavrea, B.; Gavrea, I. On some Ostrowski type inequalities. Gen. Math. 2010, 18, 33-44.

22. Hudzik, H.; Maligranda, L. Some remarks on s-convex functions. Aequationes Math. 1994, 48, 100-111. [CrossRef]

23. Hadamard, J. Étude sur les propriétés des fonctions entiéres en particulier d'une fonction considéréé par Riemann. J. Math. Pures Appl. 1893, 58, 171-215.

24. Dragomir, S.S.; Fitzpatrick, S. The Hadamard inequalities for s-convex functions in the second sense. Demonstr. Math. 1999, 32, 687-696. [CrossRef]

25. Avci, M.; Kavurmaci, H.; Özdemir, M.E. New inequalities of Hermite-Hadamard type via s-convex functions in the second sense with applications. Appl. Math. Comp. 2011, 217, 5171-5176. [CrossRef]

26. İşcan, İ. Hermite-Hadamard type inequalities for harmonically convex functions. Hacet. J. Math. Stat. 2014, 43, 935-942. [CrossRef]

27. Toplu, T.; Kadakal, M.; İşcan, İ. n-Polynomial convexity and some related inequalities. AIMS Math. 2020, 5, 1304-1318. [CrossRef]

28. Butt, S.I.; Rashid, S.; Tariq, M.; Wang, X.H. Novel refinements via n-polynomial harmonically s-type convex functions and Applications in special functions. J. Funt. Spaces 2021, 2021, 6615948 . [CrossRef]

29. Tunç, M.; Göv, E.; Şanal, Ü. On tgs-convex function and their inequalities. Facta Univ. Ser. Math. Inform. 2015, 30, 679-691.

30. Kadakal, M.; İşcan, İ. Exponential type convexity and some related inequalities. J. Inequal. Appl. 2020, 2020, 82. [CrossRef]

31. Khan, M. A.; Chu, Y.-M.; Khan, T. U.; Khan, J. Some new inequalities of Hermite-Hadamard type for s-convex functions with applications. Open Math. 2017, 15, 1414-1430. [CrossRef]

32. Özdemir, M.E.; Yildiz, C.; Akdemir, A.O.; Set, E. On some inequalities for s-convex functions and applications. J. Inequal. Appl. 2013, 333, 2-11. [CrossRef]

33. Özcan, S.; İşcan, İ. Some new Hermite-Hadamard type inequalities for s-convex functions and their applications. J. Inequal. Appl. 2019, 2019, 201. [CrossRef]

34. Korus, P. An extension of the Hermite-Hadamard inequality for convex and s-convex functions. Aequationes Math. 2019, 93, 527-534. [CrossRef]

35. Xi, B.Y.; Qi, F. Some integral inequalities of Hermite-Hadamard type for convex functions with applications to means. J. Funct. Spaces. Appl. 2012, 2012, 980438. [CrossRef]

36. Mehrez, K.; Agarwal, P. New Hermite-Hadamard type integral inequalities for the convex functions and theirs applications. J. Comp. Appl. Math. 2019, 350, 274-285. [CrossRef]

37. Sahoo, S.K.; Ahmad, H.; Tariq, M.; Kodamasingh, B.; Aydi, H.; De la Sen, M. Hermite-Hadamard type inequalities involving k-fractional operator for (h,m)-convex Functions. Symmetry 2021, 13, 1686. [CrossRef]

38. Sahoo, S.K.; Tariq, M.; Ahmad, H.; Aly, A.A.; Felemban, B.F.; Thounthong, P. Some Hermite-Hadamard-Type Fractional Integral Inequalities Involving Twice-Differentiable Mappings. Symmetry 2021, 13, 2209. [CrossRef]

39. Srivastava, H.M.; Zhang, Z.-H.; Wu, Y.-D. Some further refinements and extensions of the Hermite-Hadamard and Jensen inequalities in several variables. Math. Comput. Model. 2001, 54, 2709-2717. [CrossRef] 
40. Mohammed, P.O.; Sarikaya, M.Z.; Baleanu, D. On the generalized Hermite-Hadamard inequalities via the tempered fractional integrals. Symmetry 2020, 12, 595. [CrossRef]

41. Awan, M.U.; Noor, M.A.; Mihai, M.V.; Noor, K.I. Fractional Hermite-Hadamard inequalities for differentiable s-Godunova-Levin functions. Filomat 2016, 30, 3235-3241. [CrossRef]

42. Anastassiou, G.A. Generalised fractional Hermite-Hadamard inequalities involving m-convexity and (s, m)-convexity. Facta Univ. Ser. Math. Inform. 2013, 28, 107-126.

43. Srivastava, H.M. Fractional-order derivatives and integrals: Introductory overview and recent developments. Kyungpook Math. J. 2020, 60, 73-116.

44. Fernandez, A.; Mohammed, P.O. Hermite-Hadamard inequalities in fractional calculus defined using Mittag-Leffler kernels. Math. Meth. Appl. Sci. 2020, 44, 8414-8431. [CrossRef]

45. Sarikaya, M.Z.; Yildirim, H. On Hermite-Hadamard type inequalities for Riemann-Liouville fractional integrals. Miskolc Math Notes 2017, 17, 1049-1059. [CrossRef]

46. Mohammed, P.O.; Brevik, I. A new version of the Hermite-Hadamard inequality for Riemann-Liouville fractional integrals. Symmetry 2020, 12, 610. [CrossRef]

47. Budak, H.Ü.; Agarwal, P. New generalized midpoint type inequalities for fractional integral. Miskolc Math. Notes 2019, 20, 781-793. [CrossRef]

48. Sahoo, S.K.; Tariq, M.; Ahmad, H.; Nasir, J.; Aydi, H.; Mukheimer, A. New Ostrowski-type fractional integral inequalities via generalized exponential-type convex functions and applications. Symmetry 2021, 13, 1429. [CrossRef]

49. Mohammed, P.O. Hermite-Hadamard inequalities for Riemann-Liouville fractional integrals of a convex function with respect to a monotone function. Math. Meth. Appl. Sci. 2021, 44, 2314-2324. [CrossRef]

50. Sousa, J.V.C.; Oliveira, E.C. On the $\Psi$-Hilfer fractional derivative. Commun. Nonlinear Sci. Numer. Simul. 2018, 60, 72-91. [CrossRef]

51. Liu, K.; Wang, J.; O’Regan D. On the Hermite-Hadamard type inequality for Y-Riemann-Liouville fractional integrals via convex functions. J. Inequal. Appl. 2019, 2019, 27. [CrossRef]

52. Wu, S.; Awan, M.U.; Noor, M.A.; Noor, K.I.; Iftikhar, S. On a new class of convex functions and integral inequalities. J. Inequal. Appl. 2019, 2019, 131. [CrossRef]

53. Mohammed, P.O.; Abdeljawad, T.; Zeng, S.; Kashuri, A. Fractional Hermite-Hadamard integral inequalities for a new class of convex functions. Symmetry 2020, 12, 1485. [CrossRef]

54. Watson, G.N. A Treatise on the Theory of Bessel Functions; Cambridge University Press: Cambridge, UK, 1995.

55. Sababheh, M. Convex functions and means of matrices. arXiv 2016, arXiv:1606.08099v1. 